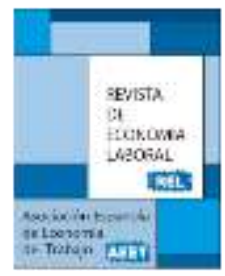

\title{
ANTES DE LLEGAR AL 13: HISTORIA Y BALANCE DE LAS JORNADAS DE ECONOMÍA LABORAL $(1995-2017)^{1}$
}

\author{
María Ángeles Davia*, Gloria Moreno** y Raúl Ramos Lobo*** \\ *UCLM, ${ }^{* *} \mathrm{UAH}$ y $* * * \mathrm{UB}$
}

Recibido Febrero 2018; Aceptado Mayo 2018

\section{Resumen}

En este trabajo se presenta un análisis de las doce Jornadas de Economía Laboral (JEL) celebradas entre 1995 y 2017. En primer lugar, se describe el nacimiento de las JEL y la evolución experimentada a lo largo de su historia. A continuación, se analizan los cambios que han tenido lugar a lo largo del tiempo en las coautorías, el idioma utilizado, la temática principal de las comunicaciones y las redes de colaboración entre autores de distintas instituciones, lo que permite obtener algunas conclusiones sobre cómo ha evolucionado la investigación en Economía Laboral en España en las tres últimas décadas.

Palabras clave: Economía Laboral, análisis bibliométrico, redes de colaboración

Clasificación JEL: J01, A11, D85

\footnotetext{
${ }^{1}$ Dada la naturaleza de este artículo, éste no ha sido sometido a evaluación por pares pero sí ha recibido comentarios del consejo editorial de la Revista de Economía Laboral, de la Junta Directiva de la AEET (Asociación Española de Economía del Trabajo) en el periodo 2017-2019 y de diversos socios entre los que destacamos a Raquel Llorente, Víctor Montuenga, Inés Murillo, Inmaculada Cebrián, Begoña Cueto, Miguel Angel Malo, Elisabet Motellón, Ignacio Perez, Héctor Sala, Hipólito Simón, Roberto Bande y Melchor Fernández así como de los participantes en las JEL 2015 donde se presentaron resultados parciales de esta comunicación. También queremos agradecer a los miembros de los comités organizadores de los workshops y las Jornadas de Economía Laboral su colaboración al compartir la información relativa a las comunicaciones presentadas, que han permitido elaborar este artículo. No obstante, sólo sus autores se responsabilizan de su contenido, las opiniones vertidas en él y los posibles errores en el manejo de la información utilizada.
}

Además, aprovechamos la ocasión para agradecer públicamente a todas las empresas y administraciones que durante todos estos años han contribuido, bien de manera regular, bien de manera esporádica, a la organización de las JEL así como al mantenimiento del Premio Lluís Fina y del programa de becas Luis Toharia. En este sentido, merecen especial mención la Fundación Santander, la Fundación Alternativas, la Fundación Juan Urrutia Elejalde y el Departamento de Economía de la Universidad de Alcalá.

(C) Revista de Economía Laboral 


\title{
Before 13: History and assessment of the Jornadas de Economía Laboral (1995-2007).
}

\begin{abstract}
This article presents an analysis of the twelve Jornadas de Economia Laboral (JEL) held between 1995 and 2017. First, we describe the origins of JEL and the evolution experience along its history. Next, we analyse the observed trends in terms of co-authorships, the language used, the topics considered in the contributions and the collaboration networks between authors of different institutions. This analysis allows us to obtain some conclusions on how the research in Labour Economics has evolved in Spain during the last three decades.
\end{abstract}

Key words: Labour economics, bibliometric analysis, collaboration networks

JEL Classification: J01, A11, D85 


\section{Algo de historia sobre las Jornadas de Economía Laboral}

El nacimiento de las Jornadas de Economía Laboral (JEL) está indisolublemente ligado a la figura de Luis Toharia. En el ámbito de la Economía Laboral su nombre no necesita presentación. Luis fue uno de los mayores expertos y conocedores del funcionamiento del mercado de trabajo español, además de un excelente profesor, dedicado y entregado a la formación de futuros economistas. Sus trabajos y publicaciones son parte de su legado y un referente para todos los investigadores y estudiosos de esta disciplina. También, a través de su participación en tareas de asesoría a diferentes Administraciones Públicas y organismos europeos nos transmitió su preocupación por conectar la academia con la realidad y la investigación con los temas relevantes para la sociedad. Es este espíritu el que, en gran medida, está detrás del origen de las JEL.

En el año 1995 Luis Toharia era profesor del Departamento de Fundamentos del Análisis Económico e Historia Económica de la Universidad de Alcalá. En aquel momento Luis había conseguido formar un grupo de investigación, mucho antes de que se implantasen de forma institucional los grupos de investigación universitarios tal y como los conocemos hoy. Se trataba de varios profesores y doctorandos que colaboraban con él habitualmente en diversos proyectos y a los que dirigía o había dirigido sus tesis doctorales.

En este contexto y como resultado de diversas conversaciones con un grupo de profesores de la Universidad del País Vasco, surge la idea de propiciar un encuentro entre investigadores del mercado laboral español, para conocer y difundir los trabajos que se estaban realizando en este ámbito. El objetivo era generar un espacio de debate e intercambio de ideas, donde poder presentar trabajos terminados o en proceso de elaboración, contando también con la presencia de representantes de diversas instituciones relacionadas con este campo, bien como productores de los datos utilizados, como destinatarios de los informes producidos o como ejecutores de las políticas laborales.

Las primeras jornadas las organizó la Universidad de Alcalá y tuvieron lugar los días 7, 8 y 9 de julio de 1995. Gracias a la implicación de Luis Toharia, al esfuerzo organizador del comité local y a la colaboración de la propia Universidad, se consiguió la financiación necesaria para facilitar la asistencia a todos los interesados, logrando que el encuentro fuera un éxito.

Dada la buena acogida de la iniciativa, los organizadores y los 
asistentes coincidieron en la conveniencia de repetir las jornadas. La Universidad del País Vasco recogió el testigo y acordó organizar las segundas JEL que se celebrarían en Bilbao dos años después. Y así, en 1997 tienen lugar las segundas jornadas que logran atraer a un número importante de autores y de ponencias. Lo que surgió inicialmente en la universidad de Alcalá como un foro de intercambio y debate, como una reunión no muy formal de amigos, conocidos e interesados en la Economía Laboral y en el mercado de trabajo español, se convirtió dos años después en unas jornadas organizadas siguiendo los cánones de cualquier congreso que se precie. Eso sí, conservando la impronta que Luis Toharia había marcado y que se ha mantenido más o menos presente hasta ahora en todas las ediciones de las jornadas.

A partir de aquí, cada dos años se han venido celebrando las JEL en distintas ciudades españolas, siempre con el respaldo de alguna universidad y de las personas que la integran. En concreto, las jornadas se han celebrado, por este orden, en Alcalá de Henares, Bilbao, Oviedo, Valencia, Reus, Alicante, Las Palmas, Zaragoza, Santiago de Compostela, Madrid, Barcelona y Valladolid. En el anexo 1 se presentan las fechas y lugares en que se han organizado las JEL en forma de cronograma mientras que en el anexo 2 se muestra información detallada sobre cada una de ellas.

Tras las jornadas celebradas en Alicante en 2005 se convocó un taller de trabajo (workshop) intermedio antes de las siguientes jornadas previstas en Las Palmas de Gran Canaria. El objetivo de esta reunión interjornadas era preparar la siguiente edición, reunir a los organizadores de las nuevas JEL con los de la última edición celebrada y al mismo tiempo recibir a ponentes que presentasen sus trabajos a un público limitado. Tal y como se muestra en el anexo 3, esta práctica se repitió en cuatro ocasiones más, pero desde 2012 no ha vuelto celebrarse ningún seminario inter-jornadas, entre otras razones por las dificultades para encontrar financiación y porque las reuniones anuales de la Junta Directiva de la AEET que también se celebraban en el marco de estos talleres pasaron a realizarse de manera telemática.

Todas y cada una de estas ediciones han sido preparadas con entrega por los organizadores, poniendo mucho trabajo y esfuerzo para conseguir no solo que el nivel académico fuese elevado, sino también que los asistentes disfrutasen de dos días intensos, incluyendo en el programa momentos de ocio y propiciando la vertiente social del encuentro más allá de las sesiones programadas. Es destacable el buen ambiente que ha caracterizado siempre a las JEL, donde los asistentes se han sentido parte 
de una "familia" y la confianza y la falta de protocolo ha facilitado que los ponentes presentasen sus trabajos sin presión de ningún tipo ante una audiencia amigable e interesada, pero también rigurosa y crítica.

La estructura de las jornadas ha ido variando según el criterio de los organizadores, pero manteniendo algunos elementos comunes en todas las ocasiones. Las ponencias admitidas se presentan en sesiones paralelas agrupadas según distintos temas. En todas las ediciones, desde la segunda, se ha venido invitando a una o varias personas de reconocido prestigio para que en sesión plenaria diesen una conferencia sobre un tema relevante y actual. Así mismo, tal y como se muestra en el anexo 4, desde las jornadas de 2001 en Valencia se ha organizado en cada congreso una mesa redonda con distintos ponentes, dirigida también a todos los asistentes. Entre los ponentes invitados se encuentran profesores universitarios, representantes de organismos españoles y extranjeros, agentes sociales y miembros de instituciones involucradas en diversos aspectos del mercado de trabajo. En las jornadas de Zaragoza en 2009 se incorporó una novedad al formato habitual, ofreciendo a los interesados un curso de formación sobre un tema de Econometría aplicada a la Economía Laboral, que se impartió el día previo al inicio de las jornadas. Tal y como se recoge en el anexo 5, el curso se ha mantenido en todas las ediciones posteriores con el mismo formato, resultando especialmente atractivo para los jóvenes investigadores que están realizando su tesis doctoral y/o comenzando su andadura profesional en este ámbito.

A lo largo de las doce ediciones de las JEL se han vivido momentos importantes que han contribuido a la continuidad de las jornadas y a afianzar su posición como congreso de Economía Laboral. En el año 2000 fallece Lluís Fina, economista, profesor de la Universidad Autónoma de Barcelona, que contribuyó de una forma muy significativa al estudio de la Economía del Trabajo, llegando a asumir puestos técnicos de la máxima relevancia tanto en la Administración central como en la Comisión Europea. Las JEL recuerdan y rinden homenaje a la figura de Lluís Fina, primero, organizando en Valencia (2001) una mesa redonda sobre la enseñanza de la Economía Laboral y después, en Reus (2003), lanzando el Premio Lluís Fina que ha alcanzado ya su octava edición, gracias a la financiación recibida en principio de la Fundación Santander y de la Fundación Alternativas y posteriormente de la Fundación Juan Urrutia Elejalde. El objetivo de este premio es mantener viva una forma de entender cómo se deben analizar los problemas del mercado de trabajo, reconociendo la excelencia en la investigación teórica o aplicada en el campo de la Economía Laboral. En cada edición de las jornadas pueden optar a él 
los trabajos publicados durante los dos años previos a la celebración de las JEL. Los premios se otorgan a partir de las deliberaciones de un jurado imparcial entre expertos del campo y se comunican y entregan en una ceremonia al final de la cena de clausura de cada edición. El anexo 5 muestra el listado de premiados en las distintas JEL.

En Reus también se presenta a los participantes de las JEL la Revista de Economía Laboral ${ }^{2}$ (REL) nacida gracias a la implicación de Luis Toharia junto con Joaquín Lorences (Oviedo), Carlos Peraita (Valencia) y Miguel Ángel Malo (USAL) y a la colaboración de la Universidad de Oviedo que inicialmente aloja en sus servidores a la revista. La REL nace con el fin de proporcionar un espacio para la publicación de artículos en el ámbito de la Economía Laboral, resultado de ponencias presentadas en las jornadas o de trabajos de investigación, en formato online y con un sistema de evaluación anónima.

En las JEL celebradas en Alicante en 2005 se crea la AEET. En una asamblea celebrada al término de las sesiones los asistentes acuerdan crear esta asociación y nombrar primer presidente a Luis Toharia. En el mismo acto se define el contenido de los estatutos que regirán la Asociación y la composición de la Junta Directiva, que se renovará cada dos años durante la celebración de las JEL. El objetivo es crear un soporte institucional que permita seguir realizando las JEL, con una Junta Directiva que asuma la función de promover y respaldar las siguientes ediciones de las jornadas y facilitar la consecución de financiación para su realización. La AEET quedará además vinculada con la REL en 2015, momento en el que comienza una nueva etapa, con nuevas secciones y monográficos anuales y con un equipo editorial compuesto por Carlos García Serrano (director) y Raquel Llorente (secretaría) en colaboración con la Junta Directiva de la Asociación.

En 2007 la AEET decide celebrar las JEL conjuntamente con las jornadas anuales de la Asociación de Economía de la Educación (AEDE) en Las Palmas de Gran Canaria. La unión de las dos jornadas resultó un éxito de asistencia y permitió encontrar lugares comunes que se plasmaron en sesiones conjuntas de ambas jornadas.

Las JEL celebradas en Santiago de Compostela en 2011 resultaron especialmente emotivas porque Luis Toharia recibió de manos del ministro Valeriano Gómez la Medalla de Oro al mérito en el Trabajo. Estas fueron sus últimas jornadas y, a pesar de lo avanzado de su enfermedad, Luis demostró una vez más su pasión por la vida, participando y disfrutando de

\footnotetext{
${ }^{2}$ La URL de la revista es como sigue: http://www.aeet-rel.es
} 
las jornadas con el buen humor, la cercanía y la calidad humana que le caracterizaban.

En esas jornadas asistieron algunos economistas italianos relacionados con la Asociación Italiana de Economistas Laborales (AIEL), iniciando así oficialmente una colaboración largamente promovida por los socios de la AEET que asistían con asiduidad a las jornadas de la AIEL, y que se mantendrá desde entonces. En las siguientes JEL se establece una sesión conjunta de la AEET y la AIEL y desde la AEET se ha alentado de forma reiterada la participación de sus socios en las conferencias de la AIEL, al tiempo que los italianos hacen lo propio, publicitando las JEL entre sus socios.

En las siguientes jornadas, en la Universidad Autónoma de Madrid (2013), ya sin Luis, se celebra una mesa redonda recordando su figura, que no deja de estar presente en el corazón y la memoria de muchos de los asistentes. En ella se presentan tanto el libro de homenaje a Luis publicado por el Ministerio de Trabajo (Jimeno y Pérez Infante, 2012) como el número monográfico de la REL de ese año, también dedicado a su recuerdo. Además, inician su andadura las becas Luis Toharia que se conceden cada edición a los participantes más jóvenes de las jornadas, financiando su asistencia. Luis demostró en todo momento un interés especial en apoyar a los que empezaban su carrera en el mundo académico e investigador; estas becas lo recuerdan y permiten que las nuevas generaciones que se incorporan a la AEET y las JEL conozcan otra dimensión de Luis, más allá de su obra publicada. De hecho, y a pesar de que no se ha recopilado información específica sobre este aspecto, sabemos que muchas de las comunicaciones presentadas en las JEL están vinculadas a un porcentaje significativo de las tesis doctorales en Economía Laboral elaboradas en nuestro país en las últimas décadas.

Desde 1995 las jornadas han experimentado cambios y han evolucionado al ritmo que marcan los tiempos. Así, por ejemplo, es evidente el proceso de internacionalización experimentado, con una mayor presencia de asistentes de fuera de España y un mayor número de ponencias escritas y presentadas en inglés, tal y como se mostrará en el siguiente apartado del trabajo. El relevo generacional, necesario para la continuidad de las jornadas, también se está produciendo, con un incremento significativo de jóvenes investigadores. Como se ha comentado, las becas Luis Toharia han jugado un papel importante en este proceso. La creación de la AEET ha posibilitado la gestión institucional de las JEL y ha facilitado el acceso a subvenciones que las financien. Todos estos cambios garantizan en buena medida la continuidad de las jornadas. 
Además de la perspectiva histórica y el análisis cualitativo realizado hasta el momento, el objetivo de este trabajo es ofrecer una aproximación a la investigación en Economía Laboral en España a través de la evolución de las JEL desde su inicio en 1995 hasta la actualidad. Para ello, en el siguiente apartado se presentan los principales resultados del análisis bibliométrico realizado en lo que se refiere a la evolución de las comunicaciones presentadas, el idioma utilizado y la temática analizada. A continuación, se describen las colaboraciones establecidas entre los autores e instituciones que han participado en las JEL a partir del análisis de redes. Por último, el trabajo concluye con algunas reflexiones sobre la evolución de la investigación en Economía Laboral en España a partir de los análisis realizados.

\section{Las comunicaciones presentadas en las JEL: evolución y temas analizados}

Entre 1995 y 2017 se han presentado en las JEL un total de 966 comunicaciones. Tal y como puede observarse en el Gráfico 2.1, el número de comunicaciones presentadas en cada una de las ediciones de las JEL ha variado considerablemente pasando de 51 en la primera edición celebrada en 1995 a 126 en la edición de 2007, que tal y como se ha comentado en la sección anterior tuvo un carácter excepcional dado que fue una edición conjunta de las JEL con las Jornadas de Economía de la Educación. En promedio se han presentado 80,5 comunicaciones por edición de las JEL (76,4 si excluimos la edición de 2007). 


\section{Gráfico 2.1. Evolución de las comunicaciones presentadas en las JEL y del número de autores.}

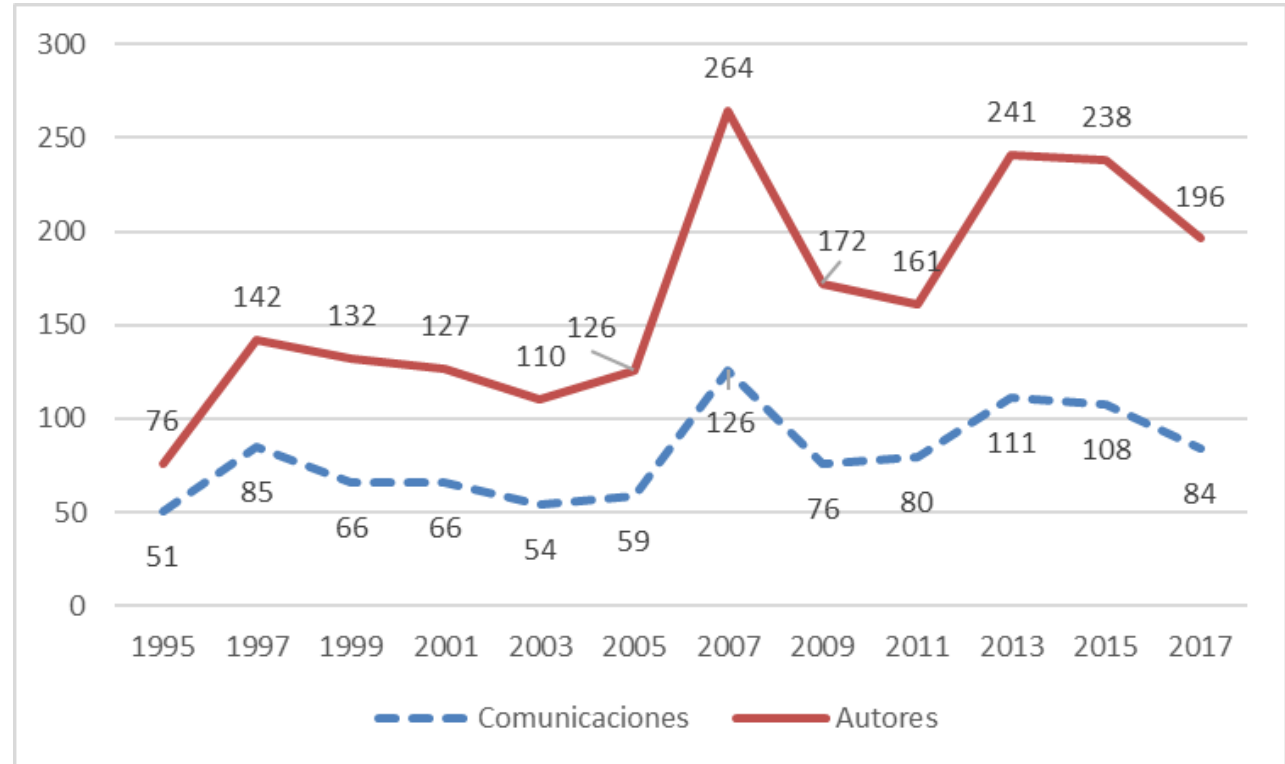

Fuente: Elaboración propia

Tal y como también puede observarse en el Gráfico 2.1, las 966 comunicaciones presentadas han sido elaboradas por 1985 autores. El hecho de que el número de autores sea superior al de contribuciones pone de manifiesto que las coautorías han sido habituales en las JEL desde sus inicios. Si analizamos el conjunto del período, el número medio de autores por comunicación es de 2,05 pero es importante destacar la tendencia creciente en este indicador que ha pasado de 1,49 en 1995 a 2,33 en 2017. De hecho, y tal como se muestra en el Gráfico 2.2, en 1995 alrededor del $60 \%$ de las comunicaciones fueron elaboradas por un único autor, mientras que, en 2017, este porcentaje se situaba por debajo del 20\%. Esta tendencia es similar a la observada en el conjunto de la investigación en economía tal y como señala Hamermesh (2013) o en el conjunto de las ciencias sociales (Tsai et al, 2016). 


\section{Gráfico 2.2. Evolución de las coautorías en las comunicaciones presentadas en las JEL.}

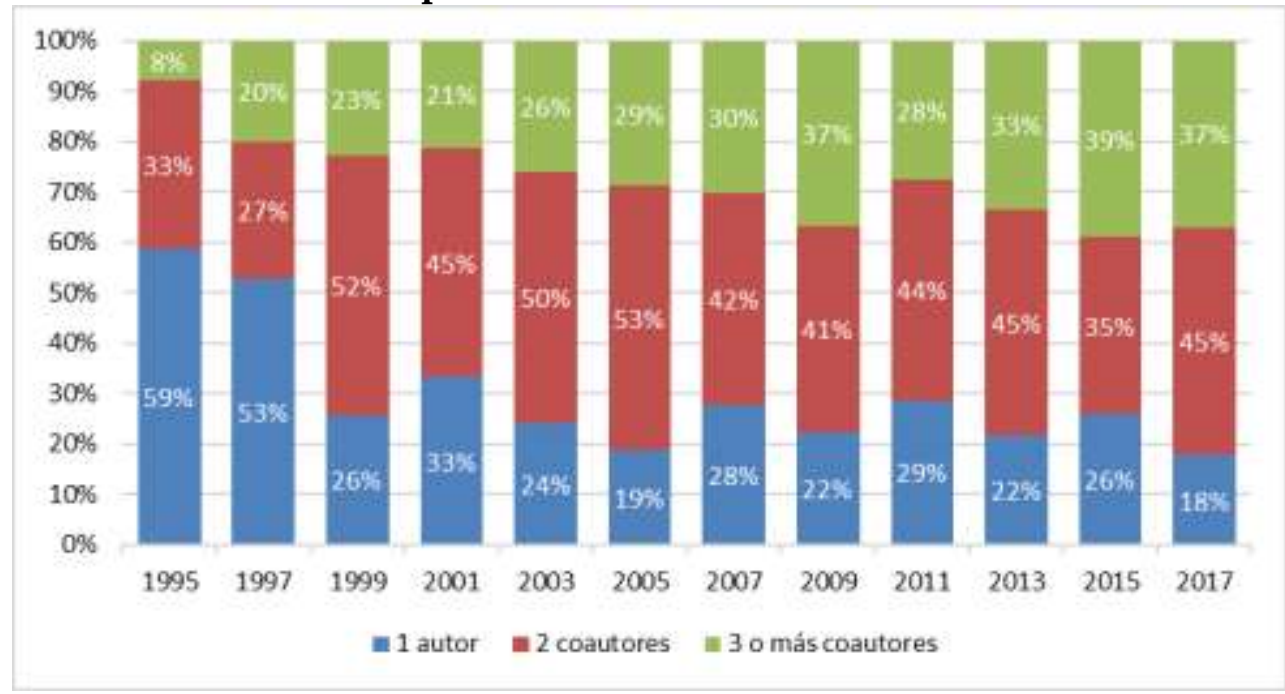

Fuente: Elaboración propia

No obstante, y a pesar del elevado número de investigadores que han participado en las comunicaciones presentadas en las JEL, hay que reconocer que una parte no desdeñable de dicha producción ha estado concentrada en un número reducido de investigadores: 33 autores, que representan un 3,5\% del total, han participado en más del $25 \%$ de las comunicaciones presentadas. Se observa, pues, una notable concentración de las contribuciones a las JEL similar a la que puede observarse en otros ámbitos como, por ejemplo, en las Reuniones de Estudios Regionales (Herranz Loncán, 2005). Esta concentración en lo que se refiere a los autores se traduce necesariamente en una situación similar en lo que se refiere a las instituciones. Las 10 instituciones de mayor producción acumulan alrededor de la mitad de las comunicaciones presentadas. De hecho, en las cuatro primeras JEL en alrededor de la mitad de las comunicaciones participaba algún investigador afiliado a la Universidad de Alcalá, Universidad del País Vasco, Universidad de Valencia y Universidad de Oviedo. Cabe destacar, sin embargo, que tanto el número de autores como instituciones distintas ha ido aumentando progresivamente a lo largo de los últimos 20 años. Tal y como se muestra en el próximo apartado, durante el período analizado han participado en las JEL investigadores de 183 instituciones distintas.

También de forma similar a la tendencia observada en el conjunto 
de la economía (y en general de la investigación científica) el inglés se ha ido imponiendo como el idioma utilizado en la redacción de las comunicaciones presentadas a las JEL tal y como se ha comentado en el apartado anterior. Si bien en las primeras jornadas la lengua mayoritaria era el español con un $80 \%$ de las comunicaciones presentadas en este idioma, este porcentaje fue decreciendo rápidamente de manera que en las JEL celebradas en 2001 la cifra de comunicaciones redactadas en español era similar al de las redactadas en lengua inglesa. En las últimas ediciones, alrededor del $60 \%$ de las comunicaciones estaban elaboradas en inglés y el $40 \%$ en español.

\section{Gráfico 2.3. Evolución de las comunicaciones presentadas en las JEL en función del idioma utilizado.}

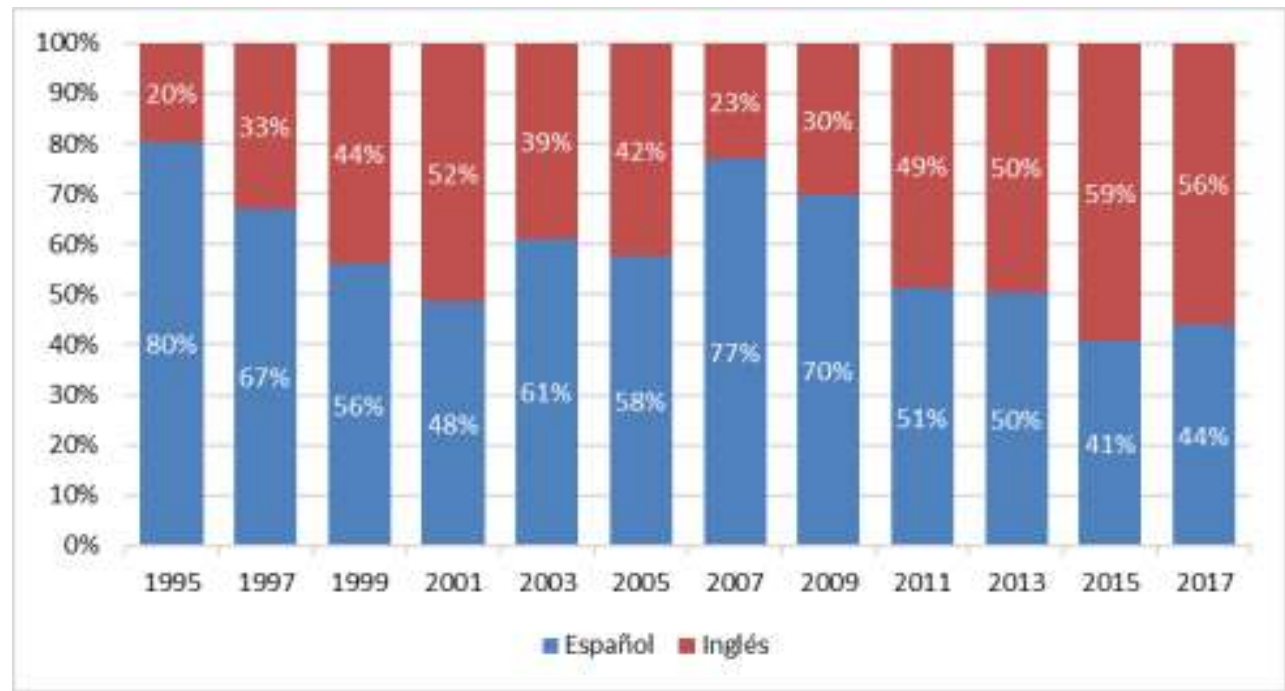

Fuente: Elaboración propia

Con el objetivo de identificar los problemas que más han preocupado a los participantes en las JEL, así como observar los cambios que se han ido produciendo en los intereses de los investigadores con el paso del tiempo, las comunicaciones presentadas se han clasificado de acuerdo con la temática principal que era objeto de análisis. Dicha temática se ha identificado a partir de las sesiones paralelas en que los distintos comités organizadores y científicos de las JEL agruparon las comunicaciones para su defensa y que mayoritariamente coincidían con la que habían solicitado los propios autores en el momento de enviar su trabajo. En este sentido, es importante destacar que ha sido necesario homogeneizar las temáticas 
consideradas en las diferentes JEL, de manera que finalmente se dispone de una clasificación en un doble nivel: el primer nivel está formado por seis temáticas principales- oferta de trabajo, demanda de trabajo, desempleo, salarios, aspectos institucionales y políticas laborales y otros temas de Economía Laboral - mientras que en el segundo nivel se consideran los siguientes quince temas: capital humano, familia, migraciones, participación, satisfacción laboral, condiciones de trabajo, empleo, temporalidad, desempleo, transiciones, diferencias salariales, discriminación y segmentación, sindicatos y negociación colectiva, aspectos institucionales y políticas laborales y otros temas de Economía Laboral.

En la tabla 2.1 se presentan los resultados de dicha clasificación para el conjunto del periodo analizado. Tal y como puede observarse en dicha tabla, la temática que ha suscitado un mayor interés entre los investigadores participantes en las JEL ha sido la relacionada con la oferta de trabajo, un tema abordado por cuatro de cada diez comunicaciones presentadas. Dentro de este ámbito, las investigaciones se han centrado mayoritariamente en aspectos relacionados con el capital humano (19\% del total). El siguiente en importancia ha sido el análisis de los salarios (21\%), seguido de la demanda de trabajo (15\%) y del desempleo (14\%).

Tabla 2.1. Comunicaciones presentadas en las JEL en función de la temática principal (1995-2017).

\begin{tabular}{|c|c|c|c|}
\hline Temática principal & \multicolumn{3}{|c|}{ Comunicaciones presentadas } \\
\hline Oferta de trabajo & 372 & $39 \%$ & \\
\hline Capital humano & 187 & & $19 \%$ \\
\hline Familia & 41 & & $4 \%$ \\
\hline Migraciones & 42 & & $4 \%$ \\
\hline Participación & 68 & & $7 \%$ \\
\hline Satisfacción laboral & 34 & & $4 \%$ \\
\hline Demanda de trabajo & 144 & $15 \%$ & \\
\hline Condiciones de trabajo & 33 & & $3 \%$ \\
\hline Empleo & 91 & & $9 \%$ \\
\hline Temporalidad & 20 & & $2 \%$ \\
\hline Desempleo & 136 & $14 \%$ & \\
\hline Desempleo & 58 & & $6 \%$ \\
\hline Transiciones & 78 & & $8 \%$ \\
\hline Salarios & 206 & $21 \%$ & \\
\hline Diferencias salariales & 95 & & $10 \%$ \\
\hline Discriminación y segmentación & 57 & & $6 \%$ \\
\hline Sindicatos y negociación colectiva & 54 & & $6 \%$ \\
\hline Aspectos institucionales y políticas laborales & 63 & $7 \%$ & \\
\hline Otros temas de Economía Laboral & 45 & $5 \%$ & \\
\hline Total comunicaciones & 966 & $100 \%$ & \\
\hline
\end{tabular}

Fuente: Elaboración propia 
En la tabla 2.2 se muestra la evolución de las temáticas consideradas a lo largo de tres subperiodos (1995-2001, 2003-2007 y 20092017) que se corresponden con distintas fases del ciclo económico y del comportamiento del mercado de trabajo español durante el período analizado. Se puede observar cómo la distribución relativa de las distintas temáticas ha cambiado a lo largo del tiempo, con un interés creciente en temas relacionados con la demanda de trabajo y un menor número de comunicaciones relacionadas con el análisis del desempleo y los salarios.

Tabla 2.2. Distribución de las comunicaciones presentadas en las JEL en función de la temática principal por subperiodos.

\begin{tabular}{lcccc}
\hline Temática principal & $\mathbf{1 9 9 5}-$ & \multicolumn{3}{c}{ Subperiodos } \\
\cline { 4 - 5 } & $\mathbf{2 0 1 7}$ & $\mathbf{1 9 9 5 - 2 0 0 1}$ & $\mathbf{2 0 0 3 - 2 0 0 7}$ & $\mathbf{2 0 0 9 - 2 0 1 7}$ \\
Oferta de trabajo & $39 \%$ & $28 \%$ & $51 \%$ & $38 \%$ \\
Demanda de trabajo & $15 \%$ & $10 \%$ & $12 \%$ & $19 \%$ \\
Desempleo & $14 \%$ & $16 \%$ & $15 \%$ & $12 \%$ \\
$\begin{array}{l}\text { Salarios } \\
\text { Aspectos institucionales y }\end{array}$ & $21 \%$ & $33 \%$ & $15 \%$ & $18 \%$ \\
políticas laborales & $7 \%$ & $5 \%$ & $6 \%$ & $8 \%$ \\
$\begin{array}{l}\text { Otros temas de Economía } \\
\text { Laboral }\end{array}$ & $5 \%$ & $7 \%$ & $0 \%$ & $5 \%$ \\
Total & & & & \\
\hline
\end{tabular}

Fuente: Elaboración propia

Un aspecto adicional a destacar es el claro vínculo entre los fenómenos que han marcado la evolución del mercado de trabajo en España en los últimos 20 años y las temáticas analizadas por los participantes en las JEL. Por ejemplo, en los Gráficos 2.4 y 2.5 se advierte una clara relación entre el número de comunicaciones presentadas en las JEL relacionadas con el desempleo y las migraciones y la evolución de ambos fenómenos en el contexto del mercado de trabajo español. 
Gráfico 2.4. Evolución de las comunicaciones presentadas en las JEL relacionadas con el desempleo y evolución de la tasa de paro de la economía española.

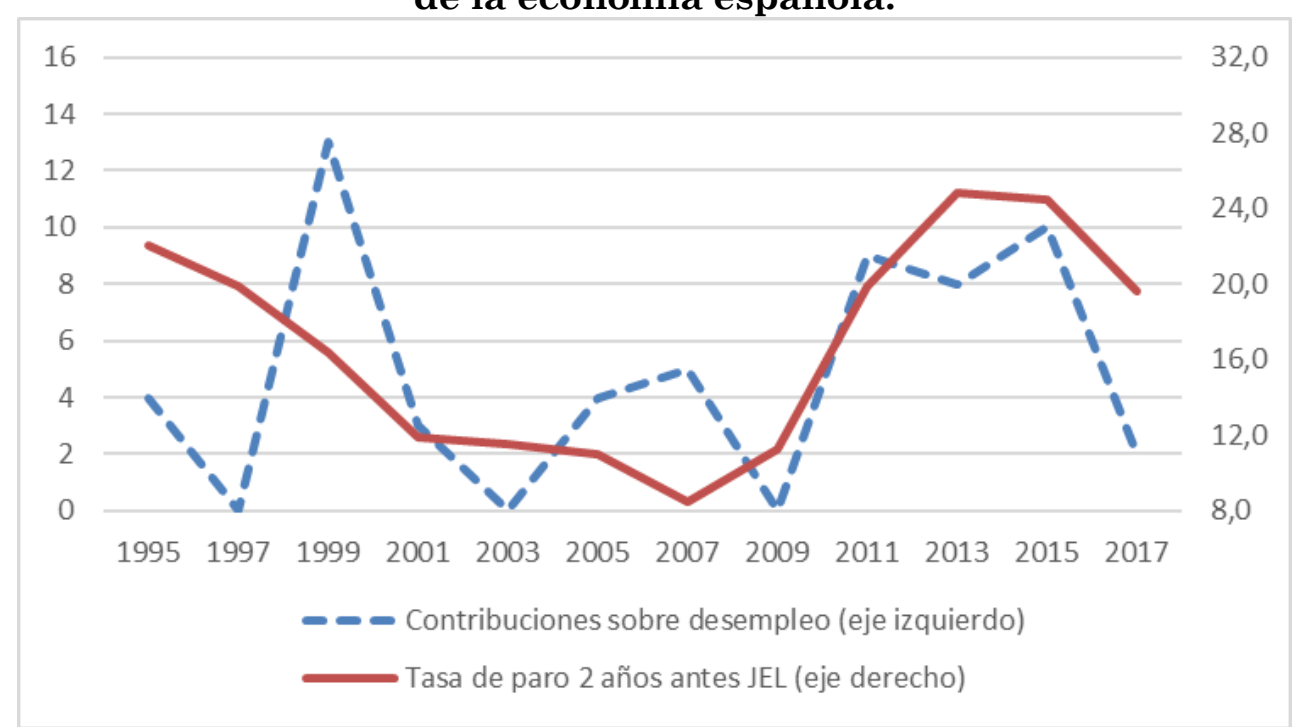

Fuente: Elaboración propia y Encuesta de Población Activa, INE

Gráfico 2.5. Evolución de las comunicaciones presentadas en las JEL relacionadas con las migraciones y evolución de las entradas migratorias en España.

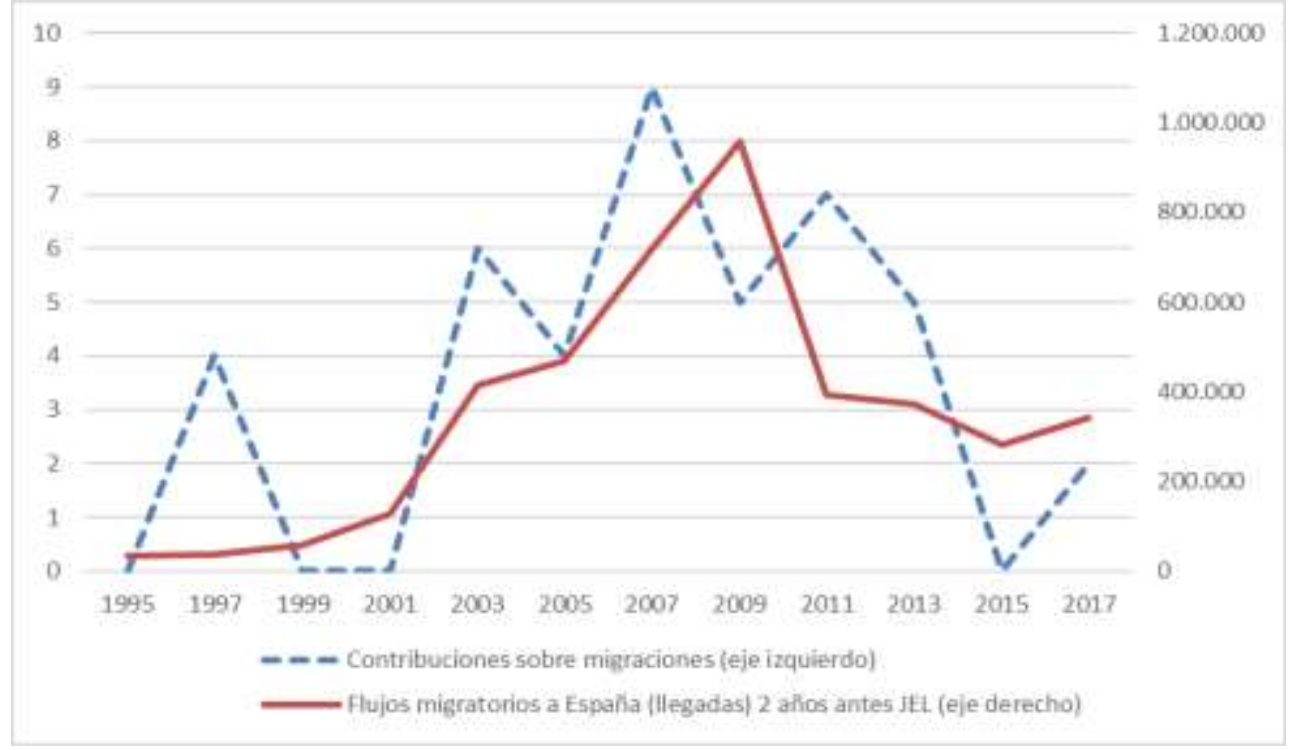

Fuente: Elaboración propia y Estadística de Migraciones, INE 


\section{Estableciendo redes de colaboración}

En el apartado anterior veíamos la evolución de las coautorías en las comunicaciones presentadas en las diferentes JEL y cómo ha ido imponiéndose el trabajo en equipos de dos o tres (muy rara vez son más) investigadores a lo largo del tiempo. A menudo las coautorías se desarrollan con compañeros de la misma institución, pero otras veces el trabajo vincula a dos y hasta tres instituciones diferentes. A este tipo de coautorías las llamaremos colaboraciones. Este apartado pretende describir las colaboraciones establecidas entre las 183 instituciones a las que están afiliados los autores de todas las comunicaciones presentadas las JEL. Esta forma de analizar colaboraciones se ha utilizado ampliamente para el estudio de las coautorías, al que dedicaremos brevemente atención. Después identificaremos y cuantificaremos las colaboraciones, describiremos la metodología utilizada (análisis de redes) y finalmente describiremos sus rasgos principales.

\subsection{Coautorias y colaboraciones en la literatura científica en España}

El análisis de redes sociales ha alcanzado bastante interés en Sociología, pero también en Geografía, Logística del transporte, Ciencias de la Computación e Inteligencia artificial (entre otras) y es esencial en Cienciometría y Bibliometría. Más que una teoría formalizada, las redes son una estrategia para conocer estructuras sociales, describiendo las relaciones entre los actores/agentes participantes e identificando la influencia que éstas pueden tener en los resultados individuales de dichos actores/agentes (Otte y Rousseau, 2002).

En Molina et al (2002) se presenta el análisis de las redes académicas como una estrategia para describir la colaboración y la estructura de influencias entre investigadores. Las redes científicas o académicas han recibido mucha atención en la literatura y tienen múltiples formas. Quizá la más antigua y extendida en la literatura es la que analiza las citas de las publicaciones, para detectar grandes líneas de investigación en las que un limitado grupo de investigadores llega a influir, directa o indirectamente, en un gran número de colegas. En esos casos el análisis de redes permite identificar estructuras de influencias académicas y relaciones jerárquicas.

La colaboración bidireccional entre académicos se inicia con contactos académicos informales (como los congresos o jornadas) y se van 
consolidando, por ejemplo, con la presentación de un seminario invitado, de modo que la forma más intensa de colaboración es la coautoría. Con frecuencia (y un poco de suerte) una coautoría se traduce en una publicación. Pero no siempre es así. Con el análisis de comunicaciones de las JEL incorporamos colaboraciones que han consolidado la red, incluso aunque no hayan terminado convirtiéndose en publicaciones académicas.

En España hay ya una abundante literatura sobre las redes de coautorías en artículos de revistas indexadas, dada la facilidad para extraer la información necesaria. En línea con lo que ocurre en otros países, estos estudios son especialmente habituales en el ámbito de las Ciencias. Por ejemplo, en Ciencias de la salud, en ámbitos como la Neurología (GonzálezAlcaide et al, 2008; Aleixandre-Benavent et al, 2013), Tabaquismo (Granda-Orive et al, 2009), Cardiología (Valderrama-Zurián et al, 2007), Farmacia Hospitalaria (Aleixandre-Benavent et al, 2008) Pediatría (Benavent et al, 2013), Biomedicina (González Alcaide et al, 2006), Autismo (Belinchón Carmona et al, 2010) y Enfermería (Almero-Canet et al, 2013). En Ciencias sociales es mucho menos frecuente: la Psicología Social (Íñiguez Rueda et al, 2006); las Ciencias de la Comunicación (FernándezQuijada, 2011); Publicidad (Baladrón-Pazos et al, 2017) y Organización y Administración de Empresas (Acedo et al, 2006). En Economía también tenemos ejemplos: Duque et al (2011) y, más recientemente, Molina et al (2016).

A diferencia de los trabajos anteriormente mencionados, el análisis que adoptamos aquí estudia la colaboración entre instituciones y no entre investigadores individuales. En esta línea destaca el trabajo de OlmedaGómez et al (2009), quienes investigan el grado de colaboración de las universidades españolas entre sí y con otras extranjeras mediante el análisis de redes sociales. Se trata de una investigación "masiva" sobre la base de todas las publicaciones que contienen la palabra "Spain" en el ámbito geográfico de la institución de alguno de los autores en grandes bases de datos bibliográficas ${ }^{3}$. Sólo seleccionando información bibliográfica de estas bases entre 2002 y 2004 parten de un universo inicial de 151.600 artículos de todo tipo, en los que al menos un autor procede de una institución de investigación española. En este estudio se detectan frecuentes colaboraciones entre universidades que se encuentran en la misma comunidad autónoma (CCAA), además de una creciente internacionalización de las colaboraciones. En consonancia con la evidencia

\footnotetext{
3 Todas ellas están integradas en Web of Science (WOS), en concreto, se trata de Science Citation Index Expanded, Social Science Citation Index y Arts and Humanities Citation Index.
} 
internacional, en la colaboración entre instituciones nacionales la proximidad geográfica es un elemento importante. Pero también lo es el pertenecer a la misma CCAA, lo que supone compartir un gobierno regional con competencias sobre la organización de las universidades ${ }^{4}$ y la distribución/asignación de fondos de investigación, que actúan como incentivos para la colaboración entre investigadores de universidades pertenecientes a la misma CCAA. Este es el caso de las universidades de Cataluña, Madrid, Valencia y Andalucía. Es en gran parte una cuestión de diseño institucional y político. En el extremo contrario, los investigadores de La Rioja, Extremadura y Castilla-La Mancha necesariamente colaboran más con universidades de fuera de la CCAA, al ser regiones con una sola universidad presencial. Estos resultados y otros que comentaremos más adelante se verán también reflejados en la pequeña red de colaboraciones que describiremos a continuación.

\subsection{Coautorias y colaboraciones en las JEL}

En este apartado cuantificaremos, en primer lugar, las colaboraciones que constituyen la unidad de análisis de las redes; en segundo lugar, describiremos las redes de colaboración conformadas por parejas de instituciones de afiliación de los autores ${ }^{5}$ de las comunicaciones presentadas en las JEL. Veremos cómo las redes se han ido ampliando y haciéndose más policéntricas, de modo que un número cada vez mayor de instituciones canalizan las posibles redes de contactos entre investigadores en Economía Laboral que participan en las JEL.

En el Gráfico 3.1 partimos de la base del porcentaje de coautorías que se pudo ver en el Gráfico 2.2, y que pasaban del 41\% en 1995 al 83\% en 2017. Las coautorías se convirtieron en la norma a partir de 1999 y, desde entonces, casi cuatro de cada cinco comunicaciones han sido en coautoría. Sobre el total de comunicaciones, las que se producen en colaboración entre dos o tres instituciones oscila entre el 13 y el $37 \%$ y, aunque no hay una

\footnotetext{
${ }^{4}$ A menudo, coautores de universidades de la misma CCAA han sido compañeros de estudios o de trabajo en el pasado y las colaboraciones surgen de equipos cuyos miembros se han disgregado, redirigiéndose hacia las instituciones más jóvenes o en expansión para consolidar sus carreras profesionales.

${ }^{5}$ En el caso de autores con varias afiliaciones se ha tomado únicamente la primera de ellas, entendiendo que es la principal. Como hay pocos autores con varias afiliaciones, esta decisión tan solo puede afectar ligeramente a la estimación del número de colaboraciones entre instituciones. Además, si un autor cambia de afiliación a lo largo del tiempo, identificaremos nuevas colaboraciones (o la interrupción de las mismas) incluso aunque no cambie de coautores.
} 
tendencia clara a lo largo del tiempo, desde 1999 representan siempre más de una quinta parte de todas las comunicaciones y entre el $30 \%$ y el $48 \%$ de las coautorías (Gráfico 3.1).

Generalmente las colaboraciones se establecen entre sólo dos instituciones, pero a veces son tres las instituciones implicadas. En el Gráfico 3.2 se representa la distribución de comunicaciones en coautoría según haya una sola institución implicada (en cuyo caso no se trataría de una colaboración entre instituciones), dos o tres instituciones. Tampoco se percibe un patrón claro en el número de instituciones que colaboran en cada comunicación y coautoría a lo largo del tiempo.

\section{Gráfico 3.1. Evolución de las colaboraciones y coautorías en las comunicaciones de las JEL.}

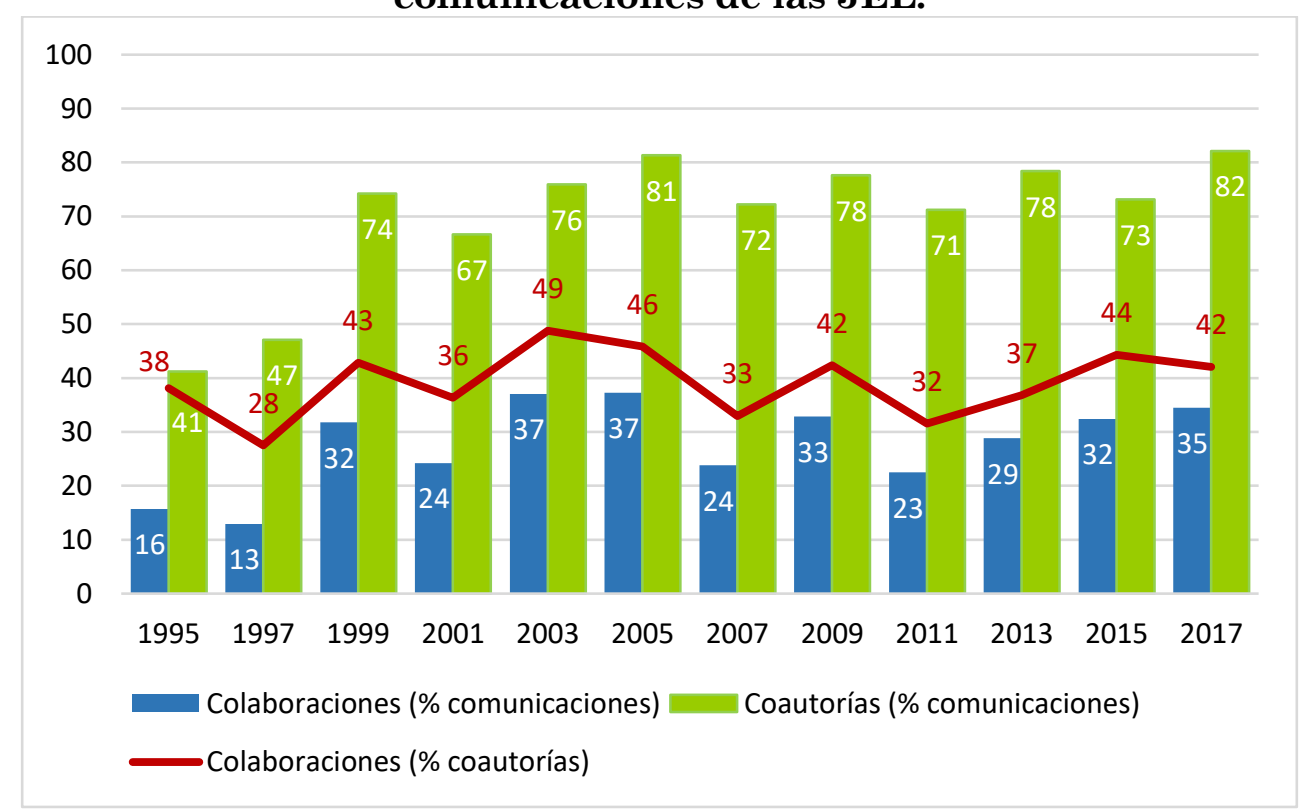

Fuente: Elaboración propia

Otra cuestión interesante a la hora de explorar las colaboraciones es la frecuencia con la que éstas se establecen entre instituciones españolas y extranjeras. Mostramos a continuación en el Gráfico 3.3 la distribución de las colaboraciones en función de la nacionalidad de las instituciones implicadas, ya excluyendo por tanto en el denominador las comunicaciones en coautoría en las que todos los autores son de la misma institución, donde por definición no hay colaboración entre instituciones. 
En el Gráfico 3.3 podemos ver que las colaboraciones con instituciones extranjeras empiezan en 1997 y desde entonces, aun siendo minoritarias, resultaban aproximadamente entre una quinta y una cuarta parte del total. Fueron algo más frecuentes en 2015, dado el marcado carácter internacional de las Jornadas de Barcelona y en 2017 más de un tercio de las colaboraciones se llevaban a cabo entre instituciones españolas y extranjeras ${ }^{6}$, lo que apunta a una internacionalización de las colaboraciones.

\section{Gráfico 3.2. Distribución de las comunicaciones en coautoría} según el número de instituciones implicadas.

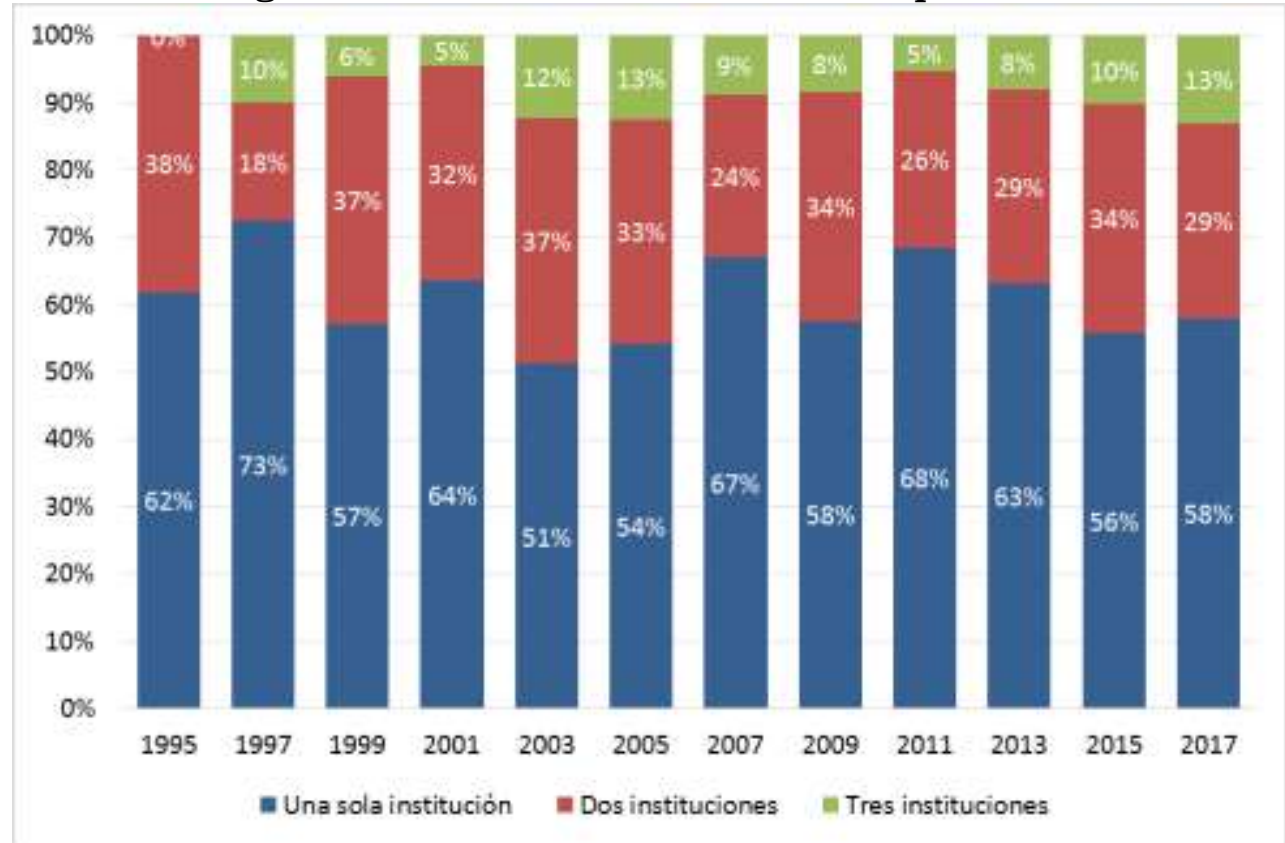

Fuente: Elaboración propia

\footnotetext{
${ }^{6}$ Aunque no se aprecia en el Gráfico 3.3, se ha observado que, en línea con las grandes tendencias detectadas en Olmeda-Gómez et al (2009), en la gran mayoría de las colaboraciones con instituciones extranjeras hay un coautor de la Unión Europea (UE), siendo Reino Unido, Italia y Francia los países comunitarios con los que se registran más colaboraciones. Fuera de la UE destacan las colaboraciones con EEUU y en Latinoamérica, principalmente con Argentina, México y Chile.
} 


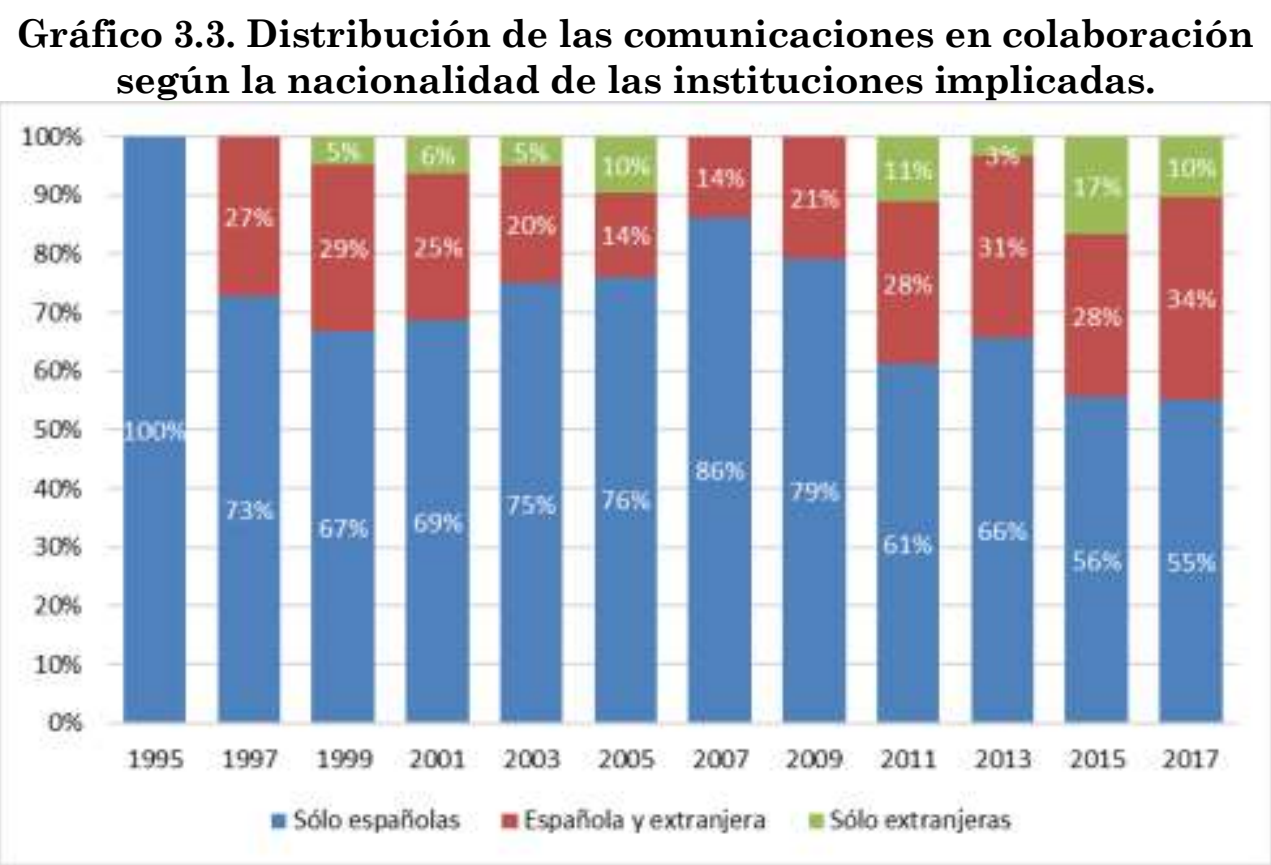

Fuente: Elaboración propia

\subsection{Análisis de redes de colaboración}

\section{Algunas precisiones terminológicas iniciales}

Las redes sociales son una aplicación de la Teoría de Grafos. Para familiarizarnos con los términos más habituales en esta herramienta metodológica nos apoyaremos en los párrafos siguientes Otte y Rousseau (2002). Una red es una estructura basada en una matriz cuadrada con ceros en la diagonal, donde todos los agentes o actores que pueden colaborar dos a dos se sitúan en filas y columnas. La unidad elemental de una red es la díada, una pareja de agentes que en nuestro caso son instituciones españolas y extranjeras a las que están afiliados los autores de todas las comunicaciones de las JEL en el periodo 1995-2017. Cada nodo de la red se representa en un punto o vértice, y los vértices están unidos por aristas ${ }^{7}$ (arcs). Los vértices pueden venir además caracterizados por algún valor

\footnotetext{
7 Resulta difícil encontrar la terminología adecuada sobre las redes en español. Aquí utilizaremos la procedente de la propuesta de Herrero (2000).
} 
que, en nuestro caso, será el número total de colaboraciones que cada nodo acumula con el resto.

Matemáticamente, un grafo $G$ consiste en una serie de nodos, que se pueden denotar como $N(G)$ y una serie de aristas $(\operatorname{arcs}), L(G)$. En la investigación sociológica los nodos se denominan actores. Cada vez que dos actores (o nodos) $i$ y $j$ establecen contacto se genera un enlace e, que representa la pareja $(i, j)$. Esta puede establecer una relación unidireccional (del $i$ al $j$ o al revés) o recíproca (grafo es no direccionado), que es el caso que nos ocupa. Cuando no están relacionados directamente entre sí, el camino desde el nodo $i$ al nodo $j$ es una secuencia de conexiones entre nodos para llegar de uno a otro. La longitud del camino de uno a otro está marcada por el número de "saltos" o contactos que hay que establecer para llegar de un actor al otro.

Las coautorías son un típico caso de relación recíproca entre actores y por tanto se representa en grafos no direccionados que se pueden resumir en una matriz simétrica $M=\left(m_{i j}\right)$ donde $m_{i j}$ adopta el valor 1 cuando hay una relación directa entre dos nodos y 0 cuando no la hay. En nuestro caso hemos sumado las colaboraciones de modo que, en nuestra matriz de relaciones, los elementos $m_{i j}$ contemplan el número de ocasiones en las que cada posible pareja de instituciones $(i, j)$ han colaborado en las JEL desde 1995 a 2017.

Las redes están caracterizadas por su nivel de cohesión global y centralidad. La cohesión se mide a través de la densidad y la centralidad es por la importancia relativa de las instituciones con más colaboraciones en el conjunto de la red. A continuación, se definen los indicadores de densidad y de centralidad.

La densidad es un indicador del grado de conectividad en el grafo y viene definida por el número de conexiones totales dividido por el número de vértices que tendría el grafo si todos los nodos estuvieran relacionados entre sí. Sus valores oscilarían entre 0 si no hubiera ninguna conexión entre instituciones y 1 si todas las instituciones estuvieran interconectadas. La centralidad es la forma de identificar la importancia de una serie de actores/nodos en la red (la frecuencia con la que se encuentran conectado con los demás, directa o indirectamente). Se define inicialmente para un actor en particular (densidad local) y se pueden obtener también en términos globales para todo el grafo. Hay tres tipos de centralidad, denominados comúnmente degree centrality, closeness centrality y betweennes centrality. En la propuesta de traducción de los términos relacionados con las redes al español en Herrero (2000) los términos equivalentes en español serían grado de centralidad, cercanía e 
intermediación.

En el grado de centralidad (degree centrality), la centralidad de un actor/nodo es igual al número de conexiones que tiene con otros actores. En nuestro caso, el grado de centralidad de una institución es el número de colaboraciones que ha tenido con otras, y se puede estandarizar dividiendo su valor (que es único para cada nodo) entre el número total de nodos con los que se podría establecer una relación ( $\mathrm{N}-1$ nodos).

El indicador de cercanía (closeness centrality) también sirve para detectar centralidad de un nodo a partir de la distancia (en el grafo) de este nodo con todos los demás. También puede ser estandarizada, pero al revés que las demás medidas de centralidad, al tratarse inicialmente de una medida de distancia más que de cercanía.

La medida de intermediación (betweenness centrality) se puede definir de forma general como el número de veces en las que un nodo necesita contactar con otro para alcanzar a un tercero. Son "saltos" necesarios para llegar de un nodo a otro atravesando otro, que tendrá una posición central. La medida de centralidad recoge el número de trayectorias más cortas que pasan por un nodo determinado. Se trata por tanto de ver la medida en que un nodo facilita la comunicación dentro de la red. Podemos decir que las instituciones con un alto grado de intermediación adoptan un papel de $h u b$ o conector/puente entre distintos grupos.

La centralidad se calcula para cada nodo y se puede obtener un valor global de la misma para la red en su conjunto, que llegaría a adoptar el valor 1 como máximo si la red tuviera forma de estrella en la que hubiera un solo nodo central y el resto de nodos estuvieran directamente (y únicamente) conectados con dicho nodo.

Los componentes de un grafo son subgrafos completamente conectados, es decir, subgrafos en los que es posible encontrar un camino entre dos nodos (Molina et al, 2002). Generalmente en una red hay varios componentes y para facilitar la visualización de la red ésta se centra en el componente principal. El resto son elementos aislados, ya sean nodos o pequeñas subredes. En nuestro análisis gráfico también reflejaremos, por simplicidad, el componente principal de la red correspondiente a cada tríada de JEL.

\section{Aplicación: las redes de colaboración en las JEL}

Para familiarizarnos con las redes de colaboración en las JEL vamos a comenzar con algunas cifras clave ya mencionadas anteriormente: de un total de 966 comunicaciones, 685 fueron en coautoría. Y de éstas, 265 se 
desarrollaron en colaboraciones entre dos (o incluso tres) instituciones diferentes. De ellas, 127 fueron colaboraciones puntuales, es decir, combinaciones de instituciones que sólo encontramos una vez. El resto de colaboraciones se han repetido dos, tres... hasta en diez ocasiones a lo largo de todo el periodo analizado. En la Tabla 3.1 mostramos las parejas de instituciones entre las que se han registrado al menos cinco colaboraciones mutuas. De las 265 colaboraciones, 71 se establecieron entre las 16 instituciones (todas ellas españolas) que aparecen en la tabla. A su vez, estas instituciones colaboraban además con otras, y sumando las colaboraciones de cada una de ellas identificamos en total 246 de las 265 colaboraciones registradas. Las instituciones que registran un mayor número de colaboraciones son la Universidad de Alcalá (UAH) con 49, la Universidad de Barcelona (UB) con 33, la Universidad Autónoma de Barcelona (UAB) con 23 y la Autónoma de Madrid (UAM) con 20. En gran medida se trata de colaboraciones cruzadas, es decir, buena parte de las colaboraciones de la UAM lo son con la UAH y en el caso de la UAB, con la UB.

El análisis de redes nos permite aprovechar toda esta información, identificando cada una de las colaboraciones entre instituciones a lo largo de la historia de las JEL, así como su frecuencia absoluta cuando se han registrado en repetidas ocasiones. Las instituciones que canalizan más colaboraciones serán importantes nodos de las redes y tendrán una gran centralidad.

Utilizando el conjunto de comandos de Stata nwcommands (Grund, 2015) hemos establecido las redes de colaboración entre las 183 instituciones a las que están afiliados los autores de las comunicaciones de todas las JEL. Los autores de 135 instituciones han trabajado en coautorías, razón por la que forman parte de posibles colaboraciones y, por tanto, se tienen en cuenta en las redes. Las 48 instituciones restantes se corresponden exclusivamente con trabajos presentados por autores que escriben en solitario, han quedado por definición descartadas para generar redes de colaboración de ningún tipo. Por tanto, trabajamos con un universo de 135 instituciones, 61 españolas y 74 extranjeras. 


\begin{tabular}{|c|c|c|c|c|c|c|c|c|}
\hline & UAH & Uniovi & Unizar & UB & UPV & US & $\begin{array}{c}\text { Total con } \\
\text { Colabora- } \\
\text { dor } \\
\text { habitual }\end{array}$ & $\begin{array}{l}\text { Total de } \\
\text { colabora- } \\
\text { ciones }\end{array}$ \\
\hline UAM & 10 & & & & & & 10 & 20 \\
\hline UAB & & & & 5 & & & 5 & 23 \\
\hline USC & & & 5 & & & & 5 & 18 \\
\hline UA & & & & 8 & & & 8 & 17 \\
\hline USAL & & 5 & & & & & 5 & 19 \\
\hline UCLM & 9 & & & & & & 9 & 16 \\
\hline UC3M & 5 & & & & & & 5 & 18 \\
\hline UCAN & & & & & 7 & & 7 & 15 \\
\hline UPO & & & & & & 7 & 7 & 15 \\
\hline UOC & & & & 10 & & & 10 & 13 \\
\hline $\begin{array}{l}\text { Total con } \\
\text { colaborador } \\
\text { habitual }\end{array}$ & 24 & 5 & 5 & 23 & 7 & 7 & 71 & 174 \\
\hline $\begin{array}{l}\text { Total } \\
\text { colabora- } \\
\text { ciones }\end{array}$ & 49 & 19 & 11 & 33 & 18 & 13 & 143 & $\begin{array}{c}246 \\
=174+143 \\
71\end{array}$ \\
\hline
\end{tabular}

Fuente: Elaboración propia

Nota: UAH - Universidad de Alcalá; UniOvi - Universidad de Oviedo; Unizar Universidad de Zaragoza; UB - Universidad de Barcelona; UPV - Universidad del País Vasco; US - Universidad de Sevilla; UAM - Universidad Autónoma de Madrid; UAB Universidad Autónoma de Barcelona; USC - Universidad de Santiago de Compostela; UA - Universidad de Alicante; USAL - Universidad de Salamanca; UCLM - Universidad de Castilla - La Mancha; UC3M - Universidad Carlos III de Madrid; UCAN - Universidad de Cantabria; UPO - Universidad Pablo Olavide; UOC - Universitat Oberta de Catalunya.

* El número total de colaboraciones detectadas entre instituciones es de 265.

La Tabla 3.2. muestra las características esenciales de la red de colaboraciones en el conjunto del periodo y para los cuatro subperiodos establecidos en el apartado anterior, cada uno de los cuales contiene tres ediciones consecutivas de las JEL. 
Tabla 3.2. Características esenciales de las redes de colaboración establecidas a lo largo de las JEL (1995-2017).

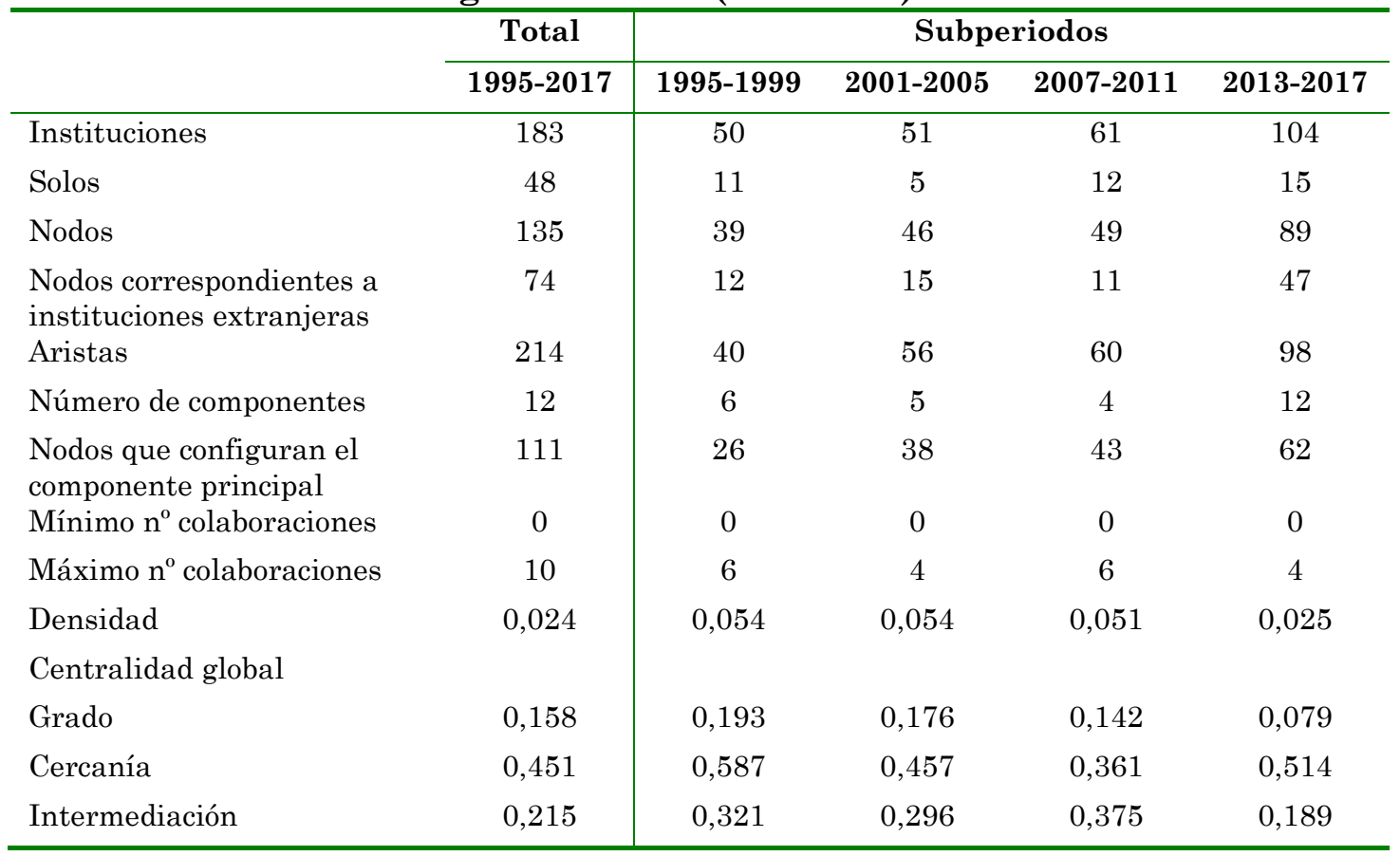

Fuente: Elaboración propia

El contenido de la Tabla 3.2. denota que el número de instituciones susceptibles de participar en colaboraciones se ha diversificado muy especialmente en la última década, duplicando las cifras iniciales hasta alcanzar las 104 en el periodo 2013-2017. En este acelerado avance final hacia la diversidad (sobre todo como resultado de la internacionalización) la red se ha hecho menos compacta y ha caído su densidad, de por sí muy baja. Se advierte que la red es menos compacta porque en el periodo final ha aumentado el número de componentes (subgrafos aislados de los demás) y el componente principal ha perdido mucho peso en la red. En cuanto a la densidad, si todas las instituciones cooperasen entre sí, la red tendría densidad 1 y sin embargo adopta valores alrededor del 0,05 hasta las JEL de 2011, para terminar en 0,25. Con la densidad también ha caído el grado de centralidad global y el de intermediación, pero no así la distancia media. Estos tres indicadores globales para la red responden a lo que ha ocurrido con los nodos (instituciones) más centrales. En la Tabla 3.3. presentamos la evolución de los indicadores de centralidad de las diez instituciones con mayor grado de centralidad a lo largo del periodo. 
Tabla 3.3. Centralidad local en las 10 universidades con mayor grado de centralidad a lo largo de las JEL (1995-2017).

\begin{tabular}{|c|c|c|c|c|c|c|}
\hline & \multirow{2}{*}{\begin{tabular}{|c|} 
Total \\
$1995-2017$
\end{tabular}} & \multicolumn{4}{|c|}{ Subperiodos } \\
\hline & & & 1995-1999 & 2001-2005 & 2007-2011 & $2013-2017$ \\
\hline \multirow[t]{3}{*}{ UAH } & Grado & 24 & 9 & 10 & 9 & 9 \\
\hline & Cercanía & 0,504 & 0,567 & 0,500 & 0,400 & 0,386 \\
\hline & Intermediación & 0,226 & 0,343 & 0,327 & 0,397 & 0,162 \\
\hline \multirow[t]{3}{*}{ UAB } & Grado & 17 & 4 & 4 & 4 & 9 \\
\hline & Cercanía & 0,453 & 0,447 & 0,321 & 0,247 & 0,421 \\
\hline & Intermediación & 0,132 & 0,097 & 0,108 & 0,138 & 0,206 \\
\hline \multirow[t]{3}{*}{ UB } & Grado & 15 & 2 & 3 & 7 & 8 \\
\hline & Cercanía & 0,450 & 0,309 & 0,391 & 0,340 & 0,389 \\
\hline & Intermediación & 0,112 & 0,034 & 0,137 & 0,172 & 0,125 \\
\hline \multirow[t]{3}{*}{ USC } & Grado & 12 & 2 & 2 & 6 & 7 \\
\hline & Cercanía & 0,425 & 0,413 & 0,375 & 0,356 & 0,411 \\
\hline & Intermediación & 0,083 & 0,000 & 0,013 & 0,355 & 0,162 \\
\hline \multirow[t]{3}{*}{ UAM } & Grado & 11 & --- & 2 & 6 & 6 \\
\hline & Cercanía & 0,421 & --- & 0,363 & 0,333 & 0,395 \\
\hline & Intermediación & 0,078 & --- & 0,000 & 0,196 & 0,142 \\
\hline \multirow[t]{3}{*}{ UniOvi } & Grado & 11 & 3 & 4 & 5 & 3 \\
\hline & Cercanía & 0,456 & 0,452 & 0,391 & 0,327 & 0,295 \\
\hline & Intermediación & 0,061 & 0,060 & 0,055 & 0,055 & 0,007 \\
\hline \multirow[t]{3}{*}{ UA } & Grado & 11 & 2 & 6 & 4 & 2 \\
\hline & Cercanía & 0,472 & 0,352 & 0,495 & 0,329 & 0,336 \\
\hline & Intermediación & 0,060 & 0,000 & 0,288 & 0,013 & 0,000 \\
\hline \multirow[t]{3}{*}{ USAL } & Grado & 9 & 1 & 5 & 4 & 4 \\
\hline & Cercanía & 0,404 & 0,418 & 0,402 & 0,324 & 0,328 \\
\hline & Intermediación & 0,035 & 0,000 & 0,099 & 0,046 & 0,035 \\
\hline \multirow[t]{3}{*}{ Uva } & Grado & 9 & -- & -- & 2 & 7 \\
\hline & Cercanía & 0,415 & -- & -- & 0,298 & 0,404 \\
\hline & Intermediación & 0,037 & --- & --- & 0,000 & 0,153 \\
\hline \multirow[t]{3}{*}{ UPV } & Grado & 9 & 3 & 6 & 2 & 1 \\
\hline & Cercanía & 0,416 & 0,365 & 0,441 & 0,284 & 0,258 \\
\hline & Intermediación & 0,051 & 0,035 & 0,149 & 0,028 & 0,000 \\
\hline
\end{tabular}

Fuente: Elaboración propia

Nota: Puede verse el significado de las siglas que identifican a cada institución al pie de la Tabla 3.1. 
En la Tabla 3.3 pueden observarse las siguientes tendencias: la primera es que el grado de centralidad de la UAH se ha mantenido siempre en primer lugar y relativamente constante, pero ha crecido al mismo tiempo el de la UAM y la UB de forma paulatina, y en las JEL de 2013 a 2017 se han incrementado con fuerza los de la UAB y la Universidad de Valladolid (UVa). Esto supone que en términos relativos la UAH perderá protagonismo en la red, como denota la evolución el indicador de intermediación. En el periodo 2013-2017 la UAH ya comparte este papel central con la UAB, la UAM y la UB que, de forma paralela, mejoran sus niveles de cercanía, al tiempo que cae el de la primera.

Una forma intuitiva de percibir la densidad y la estructura de las redes es representándolas gráficamente. El Panel 3.1. muestra los componentes principales ${ }^{8}$ de las redes correspondientes a cada tríada de jornadas consecutivas para que quede más patente su evolución en el tiempo. El grosor de los nodos denota la frecuencia de colaboraciones detectadas y los de color azul tienen en al menos uno de los extremos a una institución extranjera.

\footnotetext{
${ }^{8}$ Se trata del componente de mayor tamaño en cada subperíodo. En la Tabla 3.2 puede
} verse el número total de componentes de cada etapa. 


\section{Panel 3.1. Redes de colaboraciones entre parejas de instituciones en las JEL, 1995-2017, por subperiodos.}
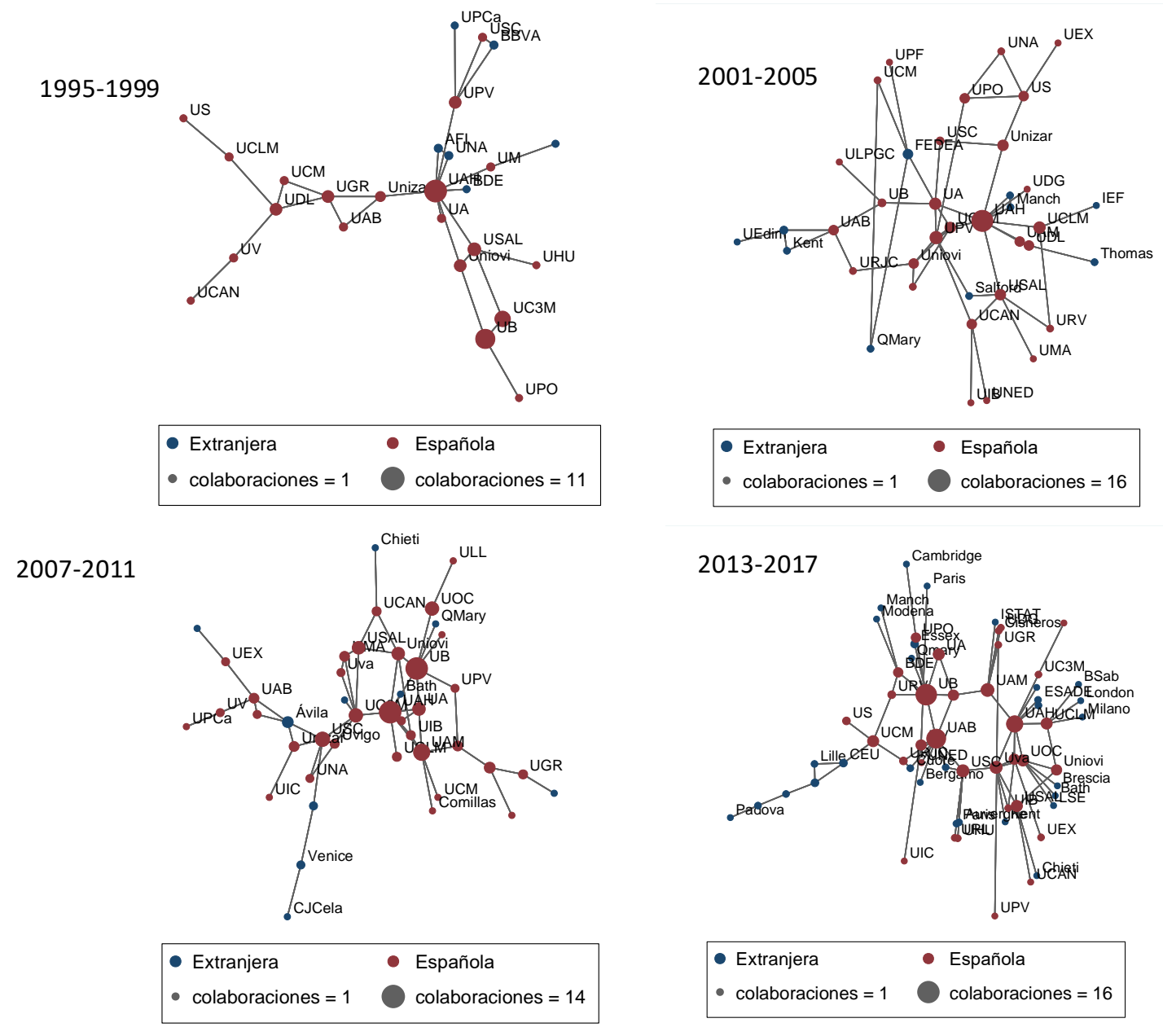

Fuente: Elaboración propia
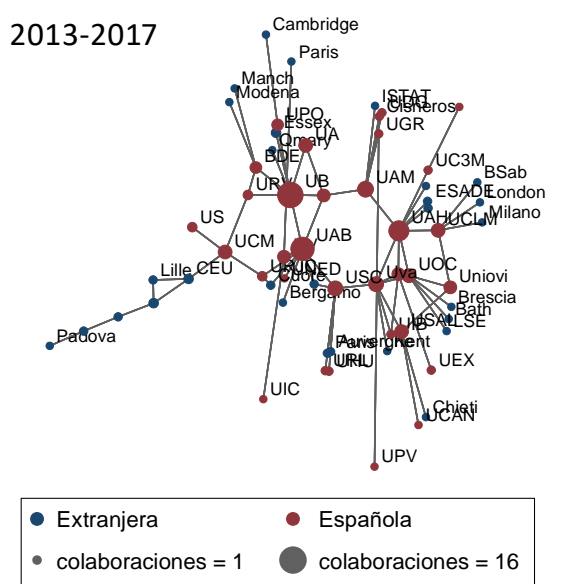

En el Panel 3.1. se puede advertir que el componente principal de la red de colaboraciones se hace cada vez más grande, que la UAH deja de ser el centro más relevante de la red y que ésta se va internacionalizando. Además, mientras que en los periodos 2001-2005 y 2007-2011 este componente principal aglutinaba entre el $80 \%$ y el $90 \%$ de los nodos, en la red del subperiodo 2013-2017 este componente apenas aglutina a dos tercios de los nodos mientras que el tercio restante se distribuye en 11 componentes adicionales, por lo que esta última red es todavía más 
compleja que la que se muestra en dicho panel.

Por tanto, podemos afirmar que, en línea con lo que se ha venido observando en la evidencia sobre estos temas (Olmeda-Gómez et al, 2009), las redes de colaboración que se han ido estableciendo entre autores de distintas instituciones en las JEL, han ido creciendo en diversidad y en internacionalización. Si bien las colaboraciones más intensas se establecen entre universidades de la misma comunidad autónoma, las redes de colaboración de las JEL son cada vez más policéntricas. La Universidad de Alcalá, donde se encuentra el germen de las JEL y de la AEET, ha ido cediendo la centralidad a otra serie de instituciones (UB, UAB, UAM) que canalizan una red cada vez más diversa y abierta a otros países.

\section{A modo de conclusión: balance y retos para el futuro}

Las JEL han ofrecido un marco de encuentro de académicos vinculados a la Economía Laboral, tanto españoles como de otros países, desde 1995. Combinan todos los aspectos que cualquier investigador puede esperar de un congreso académico: en primer lugar, es un banco de pruebas donde contrastar la validez de las propias ideas y las estrategias investigadoras cuando están en pleno desarrollo; en segundo lugar, es una escuela para investigadores en cualquier estadio de su carrera profesional: abierto a los jóvenes con cursos y premios y también a la reflexión con los más experimentados que pueden verse reconocidos a partir de la excelencia de sus publicaciones, con los agentes sociales que participan en las mesas redondas y los conferenciantes invitados. Las JEL confrontan a los participantes con la actualidad laboral, con los temas que están por investigar, se apuntan tendencias, se vislumbra el futuro y, por último, se facilita la interacción informal entre investigadores tanto a nivel académico como social contribuyendo así a la creación y consolidación de redes de investigación nacionales e internacionales en el entorno de la Economía Laboral. De hecho, las discusiones constructivas y el ambiente distendido hacen de las JEL un extraordinario entorno para el debate y el networking académico, cada vez más necesario en la Universidad española, en este mundo de redes: para la investigación, para la búsqueda de financiación, para el aprovechamiento de recursos y oportunidades, para la docencia y la formación de investigadores. El ejercicio realizado en la sección anterior muestra que la solidez de esas redes, que han ido ampliándose y evolucionando hacia un modelo multi-céntrico, cada vez más internacionalizado. 
No dudamos de que la participación en las JEL tiene un interesante rendimiento privado para los participantes: presentar trabajos en conferencias académicas supone incrementar su mayor impacto en términos de número de citas, descargas y/o lecturas (de Leon y Mcquillin, 2016). Este impacto es especialmente relevante para los investigadores más jóvenes y para aquellos que trabajan en las universidades que no se encuentran en los primeros puestos de los rankings de producción científica. Una evaluación del impacto que tiene el participar en las JEL para los trabajos presentados y sus respectivos autores contribuiría a demostrar su interés. Pero no podemos olvidar además las enormes externalidades positivas que genera, más difíciles de cuantificar $\mathrm{y}$ aprehender. La existencia de las JEL. por las redes y conexiones que ha favorecido es, por sí sola, un activo para todos, un verdadero bien público que debemos seguir aprovechando. Muchas de las acciones llevadas a cabo desde el inicio de las JEL cumplían ya con varios de los aspectos de lo que hoy en día se conoce como "Investigación e Innovación Responsables" (Responsible Research and Innovation - RRI): participación e involucración de los agentes sociales, acceso abierto, género, ética y educación científica. Aspectos que también se tendrán en cuenta en la organización de la decimotercera edición de las JEL que se celebrará en Huelva en 2019.

Este breve repaso de los orígenes y la historia de las jornadas pretende contribuir a que las próximas ediciones no pierdan la esencia de lo que han sido, un lugar de encuentro, discusión y diálogo, que posibilita el acercamiento de los investigadores del mercado laboral al propio mercado de trabajo, sus instituciones y sus agentes. Es posible que tratar de mantener la esencia inicial de las jornadas sea remar contracorriente, especialmente en un mundo académico mediatizado por los sistemas de evaluación de la investigación que priman la estandarización de la producción científica, pero merece la pena intentarlo y seguir manteniendo este espacio con sus propias señas de identidad. 


\section{Referencias bibliográficas}

Acedo, F. J., Barroso, C., Casanueva, C., \& Galán, J. L. (2006). Co-authorship in management and organizational studies: An empirical and network analysis. Journal of Management Studies, 43(5), 957-983.

Aleixandre-Benavent, R., Alonso-Arroyo, A., González de Dios, J., Sempere, Á. P., Castelló Cogollos, L., Bolaños Pizarro, M., \& Valderrama Zurián, J. C. (2013). Coautoría y redes de colaboración en la investigación española sobre esclerosis múltiple (1996-2010). Revista de Neurología, 57 (4), 157-66.

Aleixandre-Benavent, R., González-Alcaide, G., Alonso-Arroyo, A., Bolaños-Pizarro, M., Castelló-Cogollos, L., \& Valderrama-Zurián, J. C. (2008). Redes de coautorías y colaboración institucional en Farmacia Hospitalaria. Farmacia Hospitalaria, 32(4):226-337.

Almero-Canet, A., López-Ferrer, M., \& Sales-Orts, R. (2013). La colaboración interinstitucional en la producción científica española en Enfermería: análisis de redes sociales. Enfermería Clínica, 23(3), 118-127.

Baladrón-Pazos, A. J., Manchado-Pérez, B., \& Correyero-Ruiz, B. (2017). Estudio bibliométrico sobre la investigación en publicidad en España: temáticas, investigadores, redes y centros de producción (1980-2015). Revista Española de Documentación Científica, 40(2), e170. doi:http://dx.doi.org/10.3989/redc.2017.2.1411.

Belinchón Carmona, M., Boada Muñoz, L., García de Andrés, E., Fuentes Biggi, J., \& Posada de la Paz, M. (2010). Evolución de los estudios sobre autismo en España: publicaciones y redes de coautoría entre 1974 y 2007. Psicothema, 22(2): 242-249.

Benavent, R. A., de Dios, J. G., Arroyo, A. A., Pizarro, M. B., Cogollos, L. C., Alcaide, G. G., ... \& Guerrero, S. M. (2013). Coautoría y redes de colaboración científica de la pediatría española (2006-2010). Anales de Pediatría, 78(6): 410-e1-410-e11.

de Leon, F. L.L \& Mcquillin, B. (2016). The Role of Conferences on the Pathway to Academic Impact: Evidence from a Natural Experiment (November 28, 2016). SSRN: https://ssrn.com/abstract=2507361; http://dx.doi.org/10.2139/ssrn.2507361.

Duque, J.C., Ramos, R. \& Royuela, V. (2011). Research networks and scientific production in Economics: The recent Spanish Experience, Revista Ecos de Economía, A Latinamerican Journal of Applied Economics, 15 (32): 121-132.

Fernández-Quijada, D. (2011). De los investigadores a las redes: una aproximación tipológica a la autoría en las revistas españolas de comunicación. En: Piñuel Raigada, J. L.; Lozano Ascensio, C. \& García Jiménez, A. (eds.), Investigar la comunicación en España. Fuenlabrada, España: Asociación Española de Investigación de la Comunicación/Universidad Rey Juan Carlos. ISBN: 978-84-694-2713-2.

González Alcaide, G. Valderrama Zuriána, J.C., Aleixandre Benaventa, R., Alonso Arroyoa, A., De Granda Orive, J.I. \& Villanueva Serrano, S. (2006). Redes de coautoría y colaboración 
de las instituciones españolas en la producción científica sobre drogodependencias en biomedicina 1999-2004. Trastornos Adictivos, 8(2), 78-114.

González-Alcaide, G., Alonso-Arroyo, A., González de Dios, J., Sempere, A. P., ValderramaZurián, J. C., \& Aleixandre-Benavent, R. (2008). Redes de coautoría y colaboración institucional en Revista de Neurología. Revista de Neurología, 46(11), 642-51.

Granda-Orive, J. I. D., Villanueva-Serrano, S., Aleixandre-Benavent, R., ValderramaZurián, J. C., Alonso-Arroyo, A., García Río, F., C.A. Jiménez-Ruiz, S. Solano Reina \& González Alcaide, G. (2009). Redes de colaboración científica internacional en tabaquismo: análisis de coautorías mediante el Science Citation Index durante el periodo 1999-2003. Gaceta Sanitaria, 23(3), 222e34-222e43.

Grund, T. U. (2015). "nwcommands. Network Analysis in Stata". Disponible en http://nwcommands.org.

Hamermesh, D. (2013). “Six Decades of Top Economics Publishing: Who and How?" Journal of Economic Literature, 51 (1), 162-172.

Herranz Loncán, A. (2005). Una aproximación a la evolución de la ciencia regional en España a través de las contribuciones a las reuniones de estudios regionales (1973-2004), Investigaciones Regionales, 7, 219-242.

Herrero, R. (2000). La terminología del análisis de redes. Problemas de definición y de traducción. Politica y Sociedad, 33(1), 199-206.

Iñiguez Rueda, L., Muñoz Justicia, J. M., Peñaranda Cólera, M. C., \& Martínez Martínez, L. M. (2006). La psicología social en España: estructuras de comunidades. Redes: revista hispana para el análisis de redes sociales, 10 (3): 1-22.

Jimeno, J.F. y Pérez Infante, J.I. (eds) (2012): El mercado de trabajo en la obra de Luis Toharia, Colección Economía y Sociología del Trabajo. Ministerio de Empleo y Seguridad Social. Subdirección General de Información Administrativa y Publicaciones. Madrid.

Molina, J. A., Alcolea, A., Ferrer, A., Iñiguez, D., Rivero, A., Ruiz, G.\& Tarancón, A. (2016). Co-authorship and Academic Productivity in Economics: Interaction Maps from the Complex Networks Approach, IZA DP 10008.

Molina, J. L., Muñoz, J. M., \& Domènech, M. (2002). Redes de publicaciones científicas: un análisis de la estructura de coautorías. Redes: revista hispana para el análisis de redes sociales, 1(3), publicación on-line.

Olmeda-Gómez, C., Perianes-Rodriguez, A., Antonia Ovalle-Perandones, M., Guerrero-Bote, V. P., \& de Moya Anegón, F. (2009). Visualization of scientific co-authorship in Spanish universities: From regionalization to internationalization. Aslib Proceedings, 61(1): 83-100.

Otte, E., \& Rousseau, R. (2002). Social network analysis: a powerful strategy, also for the information sciences. Journal of information Science, 28(6), 441-453.

Tsai, C., Corley, E. \& Bozeman, B. (2016). Collaboration experiences across scientific 
disciplines and cohorts, Scientometrics, 108 (2), 505-529.

Valderrama-Zurián, J. C., González-Alcaide, G., Valderrama-Zurián, F. J., AleixandreBenavent, R., \& Miguel-Dasit, A. (2007). Redes de coautorías y colaboración institucional en Revista Española de Cardiología. Revista Española de Cardiología, 60(2), 117-130. 


\section{Anexos}

\section{Anexo 1. Cronograma}

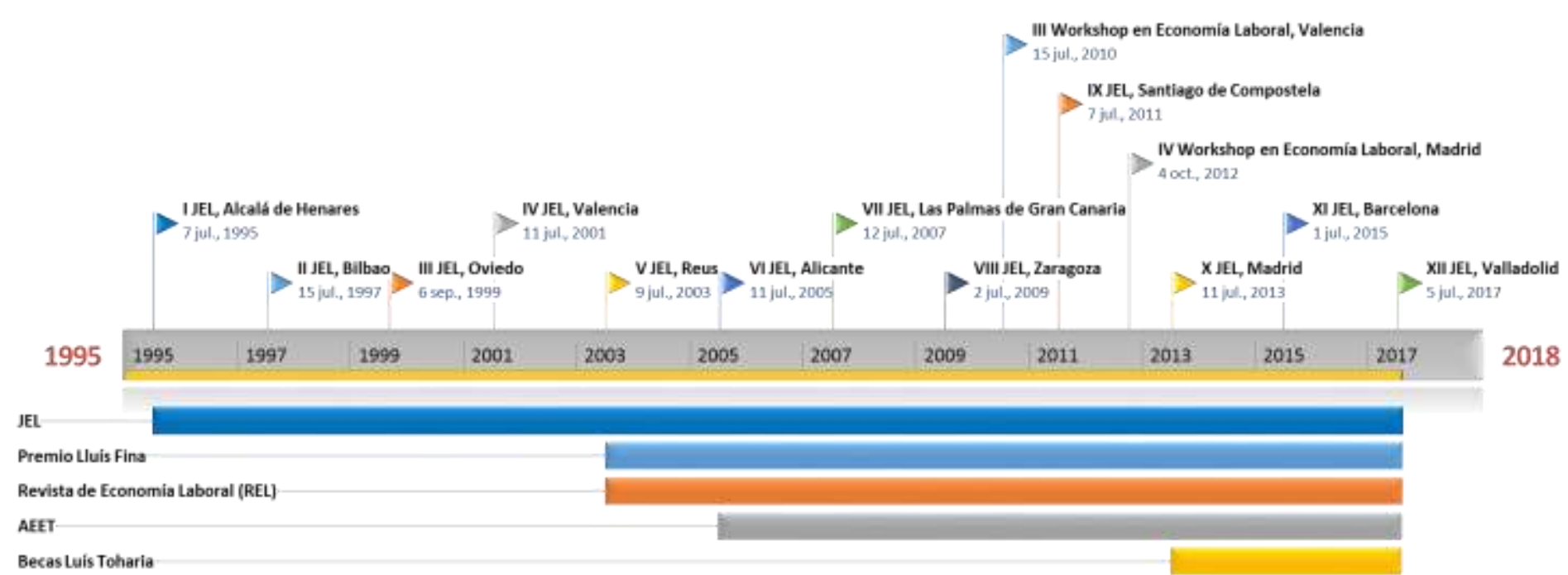

Fuente: Elaboración propia

CC Revista de Economía Laboral 


\section{Anexo 2. Jornadas de Economía Laboral 1995-2017}

\begin{tabular}{|c|c|c|c|c|c|c|}
\hline & $\begin{array}{l}\text { Lugar de } \\
\text { celebración }\end{array}$ & Universidad organizadora & $\begin{array}{l}\text { Fechas de } \\
\text { celebración }\end{array}$ & Comunicaciones & Autores & Instituciones \\
\hline I JEL & $\begin{array}{l}\text { Alcalá de } \\
\text { Henares }\end{array}$ & Universidad de Alcalá & 7-9 de Julio de 1995 & 51 (10 inglés) & 76 & 24 \\
\hline II JEL & Bilbao & Universidad del País Vasco & $\begin{array}{l}\text { 15-17 de Septiembre } \\
\text { de } 1997\end{array}$ & 85 (28 inglés) & 142 & 42 \\
\hline III JEL & Oviedo & Universidad de Oviedo & $\begin{array}{l}\text { 6-8 de Septiembre } \\
\text { de } 1999\end{array}$ & 66 (29 inglés) & 132 & 38 \\
\hline IV JEL & Valencia & Universidad de Valencia & $\begin{array}{l}\text { 11-13 de Julio de } \\
2001\end{array}$ & 66 (34 inglés) & 127 & 35 \\
\hline V JEL & Reus & Universitat Rovira i Virgili & $\begin{array}{l}\text { 9-11 de Julio de } \\
2003\end{array}$ & 54 (21 inglés) & 110 & 38 \\
\hline VI JEL & Alicante & Universidad de Alicante & $\begin{array}{l}11-13 \text { de Julio de } \\
2005\end{array}$ & 59 (25 inglés) & 126 & 39 \\
\hline $\begin{array}{l}\text { VII } \\
\text { JEL }\end{array}$ & Las Palmas & $\begin{array}{l}\text { Universidad de Las Palmas de } \\
\text { Gran Canaria }\end{array}$ & $\begin{array}{l}12-13 \text { de Julio de } \\
2007\end{array}$ & 126 (29 inglés) & 264 & 39 \\
\hline $\begin{array}{l}\text { VIII } \\
\text { JEL }\end{array}$ & Zaragoza & Universidad de Zaragoza & 2-3 de Julio de 2009 & 76 (23 inglés) & 172 & 45 \\
\hline IX JEL & $\begin{array}{l}\text { Santiago de } \\
\text { Compostela }\end{array}$ & $\begin{array}{l}\text { Universidad de Santiago de } \\
\text { Compostela }\end{array}$ & 7-8 de Julio de 2011 & 80 (39 inglés) & 161 & 43 \\
\hline X JEL & Madrid & $\begin{array}{l}\text { Universidad Autónoma de } \\
\text { Madrid }\end{array}$ & $\begin{array}{l}11-12 \text { de Julio de } \\
2013\end{array}$ & 111 (55 inglés) & 241 & 57 \\
\hline XI JEL & Barcelona & $\begin{array}{l}\text { Universitat Autònoma de } \\
\text { Barcelona }\end{array}$ & 1-3 de Julio de 2015 & 108 (64 inglés) & 238 & 73 \\
\hline $\begin{array}{l}\text { XII } \\
\text { JEL }\end{array}$ & Valladolid & Universidad de Valladolid & 5-7 de Julio de 2017 & 84 (47 inglés) & 197 & 61 \\
\hline
\end{tabular}

Fuente: Elaboración propia 


\section{Anexo 3. Workshops en Economía Laboral 2006-2012}

\begin{tabular}{llll}
\hline & Lugar de celebración & Universidad organizadora & Año de celebración \\
\hline I Workshop en Economía Laboral & Las Palmas de Gran Canaria & Universidad de Las Palmas de & 2006 \\
& & Gran Canaria & 2008 \\
II Workshop en Economía Laboral & Zaragoza & Universidad de Zaragoza & 2010 \\
III Workshop en Economía Laboral & Valencia & Universidad de Valencia & 2012 \\
IV Workshop en Economía Laboral & Madrid & Universidad Autónoma de & Madrid \\
& & & \\
\hline
\end{tabular}

Fuente: Elaboración propia 


\section{Anexo 4. Conferencias invitadas y mesas redondas celebradas en las JEL 1995-2017}

\begin{tabular}{|c|c|c|}
\hline & Conferencias invitadas & Mesas redondas \\
\hline \multicolumn{3}{|l|}{ I JEL, Alcalá de Henares } \\
\hline 1995 & & \\
\hline II JEL, Bilbao 1997 & - $\quad$ Francisco Egea (Gobierno Vasco) & \\
\hline III JEL, Oviedo 1999 & $\begin{array}{l}\text { - Richard B. Freeman (Harvard } \\
\text { University \& LSE) }\end{array}$ & \\
\hline IV JEL, Valencia 2001 & - $\quad$ Siv Gustafsson (U Amsterdam) & $\begin{array}{l}\text { - Reforma laboral, cambio tecnológico y políticas de } \\
\text { empleo - Joaquín Martínez (SERVEF), Juan Francisco } \\
\text { Jimeno (FEDEA y UAH), Carmela Martín (Funcas y } \\
\text { UCM). } \\
\text { - En memoria de Lluís Fina. La enseñanza de la Economía } \\
\text { Laboral en España. Luis Toharia (UAH), Albert Recio } \\
\text { (UAB), J. Ignacio Pérez Infante (UC3M), César Rodríguez } \\
\text { (UNIOVI) }\end{array}$ \\
\hline V JEL, Reus 2003 & $\begin{array}{ll}\text { - } & \text { Guenter Schmid } \\
\text { (Social Science Research Center) } \\
\text { - John Bishop (Cornell } \\
\text { University) }\end{array}$ & $\begin{array}{l}\text { - Retos del cambio demográfico en España: situación y } \\
\text { respuestas desde una perspectiva de mercado de trabajo. } \\
\text { Catalina Jordi (URV), Carmen de Miguel (Economista), } \\
\text { Namkee Ahn (FEDEA), M Ma Luisa Moltó (UV), Miquel Ángel } \\
\text { Purcalla (URV) }\end{array}$ \\
\hline VI JEL, Alicante 2005 & - $\quad$ Anna Cabré (CED, UAB) & $\begin{array}{l}\text { - La nueva EPA. Luis Toharia (UAH), Luis Garrido (UNED), } \\
\text { Miguel Ángel García (INE - Estadísticas Laborales y } \\
\text { Sociales) e Ignacio Pérez Infante (UC3M) } \\
\text { - Futuro y presente de la docencia Economía Laboral. César } \\
\text { Rodríguez (UNIOVI), Juan Ignacio Palacio (UCLM), J. } \\
\text { Manuel Lasierra (UNIZAR), Rafael Bonete (USAL) }\end{array}$ \\
\hline VII JEL, Las Palmas 2007 & - $\quad$ Luis Garrido Medina (UNED) & $\begin{array}{l}\text { - Inmigración y mercado de trabajo. Luis Toharia (UAH), J. } \\
\text { Ignacio Pérez Infante (UC3M), Luis Garrido Medina } \\
\text { (UNED), José Luis Rivero Ceballos (ULL) }\end{array}$ \\
\hline VIII JEL, Zaragoza 2009 & - Juan José Dolado (UC3M) & $\begin{array}{l}\text { - La crisis económica y el mercado de trabajo. Víctor } \\
\text { Montuenga (UNIZAR), Florentino Felgueroso (UNIOVI y }\end{array}$ \\
\hline
\end{tabular}




\begin{tabular}{|c|c|c|}
\hline IX JEL, Santiago 2011 & - $\quad$ Ronald Oaxaca (U Arizona) & $\begin{array}{l}\text { FEDEA), María Gutiérrez Doménech (La Caixa), José } \\
\text { Villaverde (UNICAN y FUNCAS), Melchor Fernández (USC } \\
\text { e IDEGA) } \\
\text { Retos y reformas pendientes de mercado laboral español. } \\
\text { Alberto Meixide (Director CIEF-Fundación Caixa Galicia y } \\
\text { USC), Juan Francisco Jimeno (BdE), José Ignacio Pérez } \\
\text { Infante (Ministerio de Trabajo e Inmigración), Raúl Riesco } \\
\text { (Ministerio de Trabajo e Inmigración) }\end{array}$ \\
\hline X JEL, Madrid 2013 & $\begin{array}{l}\text { - Varios ponentes, Homenaje a } \\
\text { Luis Toharia }\end{array}$ & $\begin{array}{l}\text { Desempleo juvenil y reforma del sistema educativo Maite } \\
\text { Blázquez (UAM), Cecilia Albert (UAH), Jorge Calero (UB), } \\
\text { M }^{\mathrm{a}} \text { Isabel Fernández Santos (MECD) }\end{array}$ \\
\hline XI JEL, Barcelona 2015 & $\begin{array}{l}\text { - Marco Vivarelli (U C Sacro } \\
\text { Cuore) }\end{array}$ & $\begin{array}{l}\text { - Claudio Lucifora (U C Sacro Cuore) y Rafael Domenech } \\
\text { (BBVA Research y UV) }\end{array}$ \\
\hline XII JEL, Valladolid 2017 & - $\quad$ Raymond Torres (Funcas \& ILO) & $\begin{array}{l}\text { - Juan Francisco Jimeno (Banco de España), Pedro S. } \\
\text { Martins (Queen Mary U London), Marco Leonardi (U di } \\
\text { Milano) }\end{array}$ \\
\hline
\end{tabular}

Fuente: Elaboración propia 


\section{Anexo 5. Ganadores/as del Premio Lluís Fina y cursos organizados en el contexto de las} JEL 1995-2017

\begin{tabular}{|c|c|c|}
\hline & Ganadores/as del Premio Lluís Fina & Cursos \\
\hline \multicolumn{3}{|l|}{ I JEL, Alcalá de Henares } \\
\hline \multicolumn{3}{|l|}{1995} \\
\hline \multicolumn{3}{|l|}{ II JEL, Bilbao 1997} \\
\hline \multicolumn{3}{|l|}{ III JEL, Oviedo 1999} \\
\hline \multicolumn{3}{|l|}{ IV JEL, Valencia 2001} \\
\hline \multirow[t]{2}{*}{ V JEL, Reus 2003} & I Premio Lluís Fina: & \\
\hline & Carlos García Serrano (UAH) y Miguel Ángel Malo (USAL) & \\
\hline \multirow[t]{2}{*}{ VI JEL, Alicante 2005} & II Premio Lluís Fina: & \\
\hline & Juan Ignacio Palacio (UCLM) e Hipólito Simón (UA) & \\
\hline \multirow[t]{2}{*}{ VII JEL, Las Palmas 2007} & III Premio Lluís Fina: & \\
\hline & José Ignacio García Pérez (UPO) y Yolanda Rebollo (UPO) & \\
\hline \multirow[t]{2}{*}{ VIII JEL, Zaragoza 2009} & IV Premio Lluís Fina: & Modelos microeconométricos para el análisis de la \\
\hline & $\begin{array}{l}\text { Roberto Bande (USC), Melchor Fernández (USC) y Víctor } \\
\text { Montuenga (UNIZAR) }\end{array}$ & oferta de trabajo. José $\mathrm{M}^{\mathrm{a}}$ Labeaga (IEF y UNED) \\
\hline \multirow[t]{2}{*}{ IX JEL, Santiago 2011} & V Premio Lluís Fina & The econometrics of wage decomposition, Ronald \\
\hline & Hipólito Simón (UA) & Oaxaca (U Arizona) y Yolanda Pena-Boquete (USC) \\
\hline \multirow[t]{4}{*}{ X JEL, Madrid 2013} & VI Premio Lluís Fina: & Panel data course, Raquel Carrasco (UC3M) \\
\hline & Patricia Suárez (UNIOVI), Matías Mayor (UNIOVI) y & \\
\hline & Begoña Cueto (UNIOVI). & \\
\hline & $\begin{array}{l}\text { Accésit: Elisabet Motellón (UOC), Enrique López-Bazo (UB) } \\
\text { y Mayssun El-Attar (UB) }\end{array}$ & \\
\hline \multirow[t]{2}{*}{ XI JEL, Barcelona 2015} & VII Premio Lluís Fina: & Time Series Econometrics, Luis Gil-Alaña (U Navarra) \\
\hline & $\begin{array}{l}\text { María Cervini-Plá (U Girona), Xavier Ramos (UAB) y José I. } \\
\text { Silva (U Girona) }\end{array}$ & \\
\hline \multirow[t]{2}{*}{ XII JEL, Valladolid 2017} & VIII Premio Lluís Fina: & Public interventions in labour market: Impact \\
\hline & Rodolfo Campos (IESE) y Iliana Reggio (UC3M) & evaluation designs, Ignacio Moral-Arce (AIReF) \\
\hline
\end{tabular}

Fuente: Elaboración propia 